\title{
Atmosphere Impact Losses
}

\author{
Hilke E. Schlichting ${ }^{1,2}\left(\right.$ D $\cdot$ Sujoy Mukhopadhyay ${ }^{3}$
}

Received: 15 November 2016 / Accepted: 11 January 2018 / Published online: 23 January 2018

(C) The Author(s) 2018. This article is published with open access at Springerlink.com

\begin{abstract}
Determining the origin of volatiles on terrestrial planets and quantifying atmospheric loss during planet formation is crucial for understanding the history and evolution of planetary atmospheres. Using geochemical observations of noble gases and major volatiles we determine what the present day inventory of volatiles tells us about the sources, the accretion process and the early differentiation of the Earth. We further quantify the key volatile loss mechanisms and the atmospheric loss history during Earth's formation. Volatiles were accreted throughout the Earth's formation, but Earth's early accretion history was volatile poor. Although nebular $\mathrm{Ne}$ and possible $\mathrm{H}$ in the deep mantle might be a fingerprint of this early accretion, most of the mantle does not remember this signature implying that volatile loss occurred during accretion. Present day geochemistry of volatiles shows no evidence of hydrodynamic escape as the isotopic compositions of most volatiles are chondritic. This suggests that atmospheric loss generated by impacts played a major role during Earth's formation. While many of the volatiles have chondritic isotopic ratios, their relative abundances are certainly not chondritic again suggesting volatile loss tied to impacts. Geochemical evidence of atmospheric loss comes from the ${ }^{3} \mathrm{He} /{ }^{22} \mathrm{Ne}$, halogen ratios (e.g., $\mathrm{F} / \mathrm{Cl}$ ) and low $\mathrm{H} / \mathrm{N}$ ratios. In addition, the geochemical ratios indicate that most of the water could have been delivered prior to the Moon forming impact and that the Moon forming impact did not drive off the ocean. Given the importance of impacts in determining the volatile budget of the Earth we examine the contributions to atmospheric loss from both small and large impacts.
\end{abstract}

The Delivery of Water to Protoplanets, Planets and Satellites

Edited by Michel Blanc, Allessandro Morbidelli, Yann Alibert, Lindy Elkins-Tanton, Paul Estrada, Keiko Hamano, Helmut Lammer, Sean Raymond and Maria Schönbächler

$凶$ H.E. Schlichting

hilke@ucla.edu

S. Mukhopadhyay

sujoy@ucdavis.edu

1 University of California, Los Angeles, 595 Charles E. Young Drive East, Los Angeles, CA 90095-1567, USA

2 Massachusetts Institute of Technology, 77 Massachusetts Avenue, Cambridge, MA 02139-4307, USA

3 University of California, Davis, One Shields Avenue, Davis, CA 95616, USA 
We find that atmospheric mass loss due to impacts can be characterized into three different regimes: 1) Giant Impacts, that create a strong shock transversing the whole planet and that can lead to atmospheric loss globally. 2) Large enough impactors $\left(m_{\text {cap }} \gtrsim \sqrt{2} \rho_{0}(\pi h R)^{3 / 2}\right.$, $r_{\text {cap }} \sim 25 \mathrm{~km}$ for the current Earth), that are able to eject all the atmosphere above the tangent plane of the impact site, where $h, R$ and $\rho_{0}$ are the atmospheric scale height, radius of the target, and its atmospheric density at the ground. 3) Small impactors $\left(m_{\min }>4 \pi \rho_{0} h^{3}\right.$, $r_{\min } \sim 1 \mathrm{~km}$ for the current Earth), that are only able to eject a fraction of the atmospheric mass above the tangent plane. We demonstrate that per unit impactor mass, small impactors with $r_{\text {min }}<r<r_{\text {cap }}$ are the most efficient impactors in eroding the atmosphere. In fact for the current atmospheric mass of the Earth, they are more than five orders of magnitude more efficient (per unit impactor mass) than giant impacts, implying that atmospheric mass loss must have been common. The enormous atmospheric mass loss efficiency of small impactors is due to the fact that most of their impact energy and momentum is directly available for local mass loss, where as in the giant impact regime a lot of energy and momentum is 'wasted' by having to create a strong shock that can transverse the entirety of the planet such that global atmospheric loss can be achieved. In the absence of any volatile delivery and outgassing, we show that the population of late impactors inferred from the lunar cratering record containing $0.1 \% M_{\oplus}$ is able to erode the entire current Earth's atmosphere implying that an interplay of erosion, outgassing and volatile delivery is likely responsible for determining the atmospheric mass and composition of the early Earth. Combining geochemical observations with impact models suggest an interesting synergy between small and big impacts, where giant impacts create large magma oceans and small and larger impacts drive the atmospheric loss.

Keywords Volatiles $\cdot$ Accretion $\cdot$ Planet formation $\cdot$ Water $\cdot$ Impacts

\section{Introduction}

Terrestrial planet formation can generally be characterized by three distinct stages. The first is dominated by the accretion of planetesimals, which results in the formation of planetary embryos comparable to their isolation masses. The isolation mass is simply the mass that a planetary embryo can grow to by locally accreting all the material available in its feeding zone and it is given by

$$
M_{\text {iso }}=\frac{\left(10 \pi \Sigma a^{2}\right)^{3 / 2}}{\left(3 M_{\odot}\right)^{1 / 2}},
$$

where $\Sigma$ is the disk surface density in solids, $a$ the semi-major axis and $M_{\odot}$ the mass of the sun (e.g. Ida and Makino 1993; Weidenschilling et al. 1997; Schlichting 2014). Evaluating the isolation mass, assuming that $\Sigma$ is given by the minimum-mass solar nebular (MMSN), $\Sigma_{M M S N} \simeq 10 \times(a / 1 \mathrm{AU})^{-3 / 2} \mathrm{~g} \mathrm{~cm}^{-2}$ (Hayashi 1981), yields at $1 \mathrm{AU} M_{\text {iso }} \sim M_{\text {Mars }}$. This implies that Mars may indeed have formed and survived as an isolation mass, but that Earth and Venus must have undergone an additional stage of accretion. The second stage consists of a series of giant impacts of a few dozen embryos that merge to form the Earth and Venus (e.g. Agnor et al. 1999; Chambers 2001), with the Moon forming impact being the last giant impact of the Earth (e.g. Cameron and Benz 1991; Canup and Asphaug 2001; Ćuk and Stewart 2012). Giant impacts begin when the planetesimals are no longer able to efficiently damp the eccentricities that are mutually excited by the growing planetary embryos. Order of magnitude estimates that balance the stirring rates of the protoplanets with the damping 
rates due to dynamical friction by the planetesimal population and numerical simulations find that giant impacts set in when the total mass in protoplanets is comparable to the mass in planetesimals (Goldreich et al. 2004; Kenyon and Bromley 2006; Schlichting et al. 2012). This implies that about $50 \%$ of the total mass still resides in planetesimals when giant impacts begin and planetesimal accretion continues throughout the giant impact phase and afterwards. Furthermore, there exists geochemical evidence that a total of about $0.01 M_{\oplus}$ of chondritic material was delivered as 'late veneer' by planetesimals to the terrestrial planets after the end of giant impacts (Warren et al. 1999; Walker et al. 2004; Walker 2009), which is the third stage of accretion. Such late veneer, if accreted in small planetesimals, has the additional advantage that it can damp, due to the absence of strong mutual excitation among the terrestrial planets by the end of the impact stage, the post giant impact eccentricities to the almost circular values that we observe today (Schlichting et al. 2012). The terrestrial planets therefore had a rich and diverse formation history with volatiles being delivered and eroded during the planets' accretion of small planetesimals and mergers between large planetary embryos. As a result, in order to understand the origin and evolution of the terrestrial planets' atmospheres one needs to examine the contribution to volatile delivery and loss by both the giant impacts and planetesimal accretion. Geochemical observations of noble gases and major volatiles determine what the present day inventory of volatiles tells us about their sources and their accretion process as well as the key volatile loss mechanisms during Earth's formation. In this chapter we combine geochemical observations and models of small planetesimal impacts and giant collisions to shed light on the volatile delivery and atmospheric loss history of the Earth. We show that the data and models point to a rich interplay between volatile accretion and atmospheric mass loss facilitated by small and large impacts. This chapter is structured as follows: Earth's geochemical constraints on the delivery of its volatiles and losses are discussed in Sect. 2. Analytical impact models ranging from small planetesimal impacts to giant impacts are presented in Sect. 3 and used to calculate the atmospheric mass loss during accretion and the delivery of the late veneer highlighting the importance and efficiency of various different impact regimes. Conclusions follow in Sect. 4.

\section{Volatile Delivery to and Losses from the Earth: Constraints from Observations}

The relative abundances of a suite of elements $(\mathrm{H}, \mathrm{C}, \mathrm{N}$, halogens, noble gases) along with their isotopic compositions allow us to decipher where volatiles were derived from during Earth's accretion. Measurements in mantle-derived rock, combined with volatile abundances in the Earth's exosphere, are now providing us with insights into both the total abundance of volatiles and their initial distribution on the Earth. Recent measurements are also providing key new information on the importance of processes like hydrodynamic escape and impacts in shaping the final abundance of the volatile budget for Earth (Marty 2012; Halliday 2013; Tucker and Mukhopadhyay 2014; Sharp and Draper 2013; Hirschmann 2016).

\subsection{Volatile Sources and the History of Volatile Delivery}

Over the past fifteen years, the non-radiogenic neon $(\mathrm{Ne})$ isotopes $\left({ }^{20} \mathrm{Ne} /{ }^{22} \mathrm{Ne}\right)$ have played an important role in providing constraints on the acquisition of volatiles. Earth could have acquired $\mathrm{Ne}$ from potentially three sources: i) nebular $\mathrm{Ne}$ with ${ }^{20} \mathrm{Ne} /{ }^{22} \mathrm{Ne}$ ratio of $13.36 \pm 0.09$ (Heber et al. 2012); ii) micron-sized dust grains irradiated by solar wind in the 

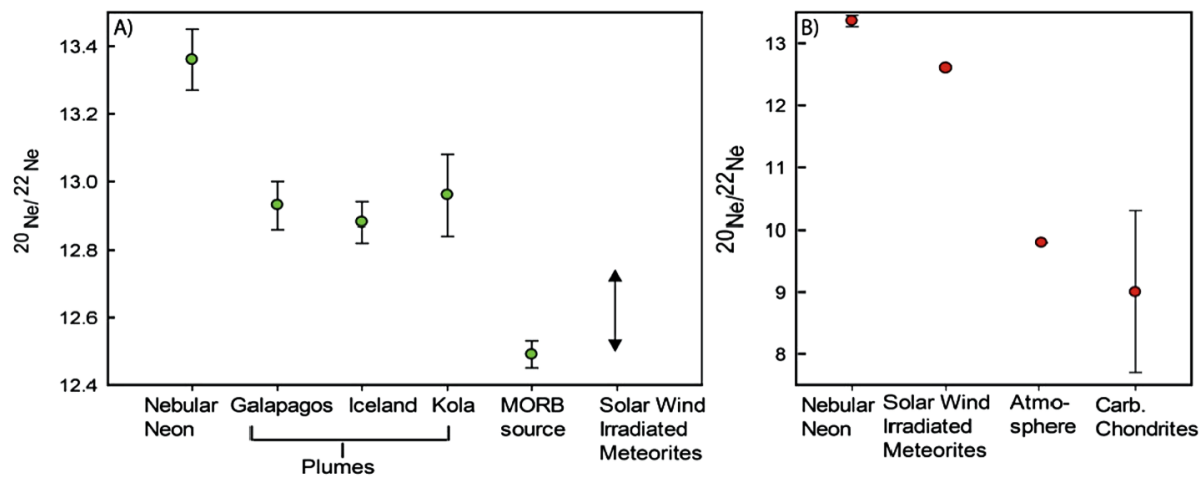

Fig. 1 The Neon isotopic composition of the mantle and atmosphere compared with that of the solar nebula, solar wind irradiated material and CI chondrites. The nebular value is from Heber et al. (2012), the range of solar wind irradiated material is from Moreira and Charnoz (2016) and the CI chondrite data is from Mazor et al. (1970). The Galapagos, Iceland and Kola plume points represent the highest measured values from these plumes and are from Péron et al. (2016), Mukhopadhyay (2012) and Yokochi and Marty (2004), respectively. The MORB source is from Holland and Ballentine (2006). So far, MORBs removed from the influence of plumes have not yielded ${ }^{20} \mathrm{Ne} /{ }^{22} \mathrm{Ne}$ ratios that are statistically different from 12.5. The large error bars for the $\mathrm{CI}$ chondrites reflect the inherent heterogeneity in $\mathrm{Ne}-\mathrm{Ar}$ composition within this meteorite class. Error bars in both panels are 1 sigma and the large error bars for the CI chondrities reflect the inherent heterogeneity in Ne composition within this meteorite class

early Solar System and then incorporated into planetesimals $\left({ }^{20} \mathrm{Ne} /{ }^{22} \mathrm{Ne}\right.$ of $\sim 12.5$ to 12.7 , although higher values might be possible if irradiation is not in steady state with sputtering; Moreira and Charnoz (2016)) and iii) $\mathrm{Ne}$ from carbonaceous chondrites $\left({ }^{20} \mathrm{Ne} /{ }^{22} \mathrm{Ne}\right.$ of $\sim 8$ ). The shallow mantle that is most frequently tapped during the mid ocean ridge volcanism (MORB source) appears to have a ${ }^{20} \mathrm{Ne} /{ }^{22} \mathrm{Ne}$ of $12.49 \pm 0.04$, similar to solar wind irradiated material that is found in gas-rich meteorites (Fig. 1; Trieloff et al. (2000), Ballentine et al. (2005), Holland and Ballentine (2006)). However, the deep mantle, sampled by plumes, appear to reach ${ }^{20} \mathrm{Ne} /{ }^{22} \mathrm{Ne}$ values close to 13.0 (Sarda et al. 2000; Yokochi and Marty 2004; Mukhopadhyay 2012; Péron et al. 2016). This value is higher than expected from solar wind irradiation of grains and significantly higher than values in carbonaceous chondrites raising the intriguing possibility that within the deepest mantle, there is a memory of Earth's acquisition of nebular volatiles.

The presence of nebular volatiles in the deep mantle plume source suggests that volatile accretion started in the presence of nebular gases while the Earth was an embryo. While nebular gases can persists for $\sim 10$ Myrs, the median lifetime of a nebula, i.e. the time at which half of all systems lost their gas disks, is $\sim 2.5$ Myrs (Hillenbrand 2008). The mean time of accretion for Earth (i.e. the time to have grown to $50 \%$ of the final mass) is $\sim 11$ Myrs (Yin et al. 2002) while the mean accretion timescale of Mars is $1.9_{0.8}^{+1.7}$ Myrs (Dauphas and Pourmand 2011; Tang and Dauphas 2014). Clearly, the entire planet could not have equilibrated with the solar nebula. However, given a median lifetime of 2.5 Myrs for the nebula, a relatively high probability event would be for one or more embryos incorporated into Earth, 1-3 times the size of Mars, to have acquired nebular volatiles.

With the exception of the deep mantle plume source, signatures of nebular gases are not found elsewhere on our planet. Ne isotopes in the MORB source are similar to solar wind irradiated meteoritic sources (e.g. Ballentine et al. 2005; Moreira 2013) and the isotopic ratios of $\mathrm{H}, \mathrm{N}$, argon (Ar), krypton $(\mathrm{Kr})$ and xenon $(\mathrm{Xe})$ in the convecting MORB mantle source and in the atmosphere (with the exception of $\mathrm{Xe}$ ) are most similar to chondritic meteorites 
(Alexander et al. 2012; Marty 2012; Halliday 2013; Hirschmann 2016). Furthermore, even the deep mantle values are not identical to nebular values. These observations suggest that most of the nebular volatiles acquired by the Earth embryos must have been lost and volatile elements were later replenished through delivery of chondritic volatiles after the dissipation of the nebula. We note that the isotopic compositions of the major volatiles rule out comets as the dominant source of Earth's major volatile species since these icy bodies are enriched in the heavier isotopes of $\mathrm{H}$ and $\mathrm{N}$ compared to Earth (Bockelée-Morvan et al. 1998; Meier et al. 1998; Jehin et al. 2009; Halliday 2013; Marty 2012; Marty et al. 2016)

The loss of nebular volatiles from within the Earth during its embryo stage may be a necessary consequence of the continued accretion of the Earth. After the nebula dissipates, the Earth embryo grows through impact of planetesimals and through giant impacts associated with collisions between embryos, with the Moon forming giant impact effectively terminating Earth's accretion. Since magma oceans are a consequence of giant impacts, the collisions between embryos would likely have released a large fraction of gases trapped within the solid Earth into the atmosphere. Hydrodynamic escape following the dissipation of the nebular and/or subsequent impacts must have led to loss of these nebular volatiles from the Earth embryo. Alternatively, some of the volatile elements may have been sequestered in the core (e.g. Dasgupta et al. 2013; Hirschmann 2016).

The timing of acquisition of volatiles is currently still a subject of debate (Marty 2012; Halliday 2013; Tucker and Mukhopadhyay 2014; Hirschmann 2016). The two possible hypothesis are that the majority of these volatiles were delivered prior to the Moon forming impact, or were delivered associated with the late accretion, defined as the last $0.3-1 \%$ of the Earth's mass that was accreted after the formation of the Moon (e.g. Warren et al. 1999; Walker 2009).

Radioactive isotopes, like ${ }^{129} \mathrm{I}$ and ${ }^{244} \mathrm{Pu}$, can provide constraints on the history of volatile accretion. I is a volatile element while $\mathrm{Pu}$ is a refractory lithophile element. While the delivery rate of $\mathrm{Pu}$ should be time invariant during terrestrial accretion, I delivery would have changed with time. Thus, the I/Pu ratio of the Earth can potentially be used as a tracer of heterogeneous volatile delivery. If mantle domains have kept a memory of the different periods of terrestrial accretion, then the heterogeneous delivery of I with respect to other refractory lithophile elements could be interrogated through the ${ }^{129} \mathrm{Xe} /{ }^{136 *} \mathrm{Xe}_{P u}$ ratio of mantle-derived basalts. Here, ${ }^{129} \mathrm{Xe}$ is produced from ${ }^{129} \mathrm{I}$ decay only during the first 100 Myrs of solar system history and ${ }^{136 *} \mathrm{Xe}_{P u}$ is ${ }^{136 *} \mathrm{Xe}$ produced from ${ }^{244} \mathrm{Pu}$ fission during the first 500 Myrs of solar system history. The ${ }^{129} \mathrm{Xe} /{ }^{136 *} \mathrm{Xe}_{P u}$ ratio has been used to constrain the closure time for volatile loss of a mantle reservoir (Pepin and Porcelli 2006; Avice and Marty 2014). Since ${ }^{129} \mathrm{I}$ has a shorter half-life than ${ }^{244} \mathrm{Pu}$, for reservoirs with the same initial $\mathrm{I} / \mathrm{Pu}$ ratio, higher ${ }^{129} \mathrm{Xe} /{ }^{136 *} \mathrm{Xe}_{P u}$ ratios are indicative of earlier closure to volatile loss. The ${ }^{129 *} \mathrm{Xe} /{ }^{136 *} \mathrm{Xe}_{P u}$ of the plume source mantle varies between $2.1 \pm 1.6$ and $2.9_{-0.1}^{+0.4}$ and the corresponding values for MORBs are between $7.8_{-1.8}^{+3.1}$ and $9.4_{-2.7}^{+5.5}$ (Fig. 2, Mukhopadhyay (2012), Petô et al. (2013), Parai and Mukhopadhyay (2015), Caracausi et al. (2016)). If the mantle had a homogeneous $\mathrm{I} / \mathrm{Pu}$ ratio, the higher ${ }^{129 *} \mathrm{Xe} /{ }^{136 *} \mathrm{Xe}_{P u}$ ratio in the MORB source would paradoxically imply that the shallow mantle became closed to volatile loss earlier than the deep mantle. A simpler explanation is that the lower ${ }^{129 *} \mathrm{Xe} /{ }^{136 *} \mathrm{Xe}_{P u}$ reflects a lower initial I/Pu ratio for the plume source compared to the MORB source. These differences would indicate that the initial phase of Earth's accretion was volatile poor compared to the later stages of accretion. This conclusion is consistent with $\mathrm{Pd}-\mathrm{Ag}$ systematics (Schönbächler et al. 2010) with the added distinction that the differences in the volatile accretion history are still preserved in the Xe isotopic composition of the mantle. As a result, the magma oceans formed during the giant impact stage of accretion did not homogenize chemical signatures acquired during the embryo stage. 
Fig. 2 Ratios of I- to Pu-derived $\mathrm{Xe}$ in plume source and MORB source assuming a chondritic starting composition for $\mathrm{Xe}$ isotopes in the mantle (Caracausi et al. 2016). The plume average was compiled from data presented in Mukhopadhyay (2012), Pető et al. (2013), and Caracausi et al. (2016). The MORB average is from Parai and Mukhopadhyay (2015)

Fig. 3 Relative terrestrial abundances of $\mathrm{C}, \mathrm{N}, \mathrm{H}$, and halogens normalized to the chondritic relative abundances. $\mathrm{H}, \mathrm{C}$ and $\mathrm{N}$ data are from Marty (2012) and Halliday (2013). Cl data is from Sharp and Draper (2013) and F data are from McDonough (2003) and Palme and O'Neill (2014)
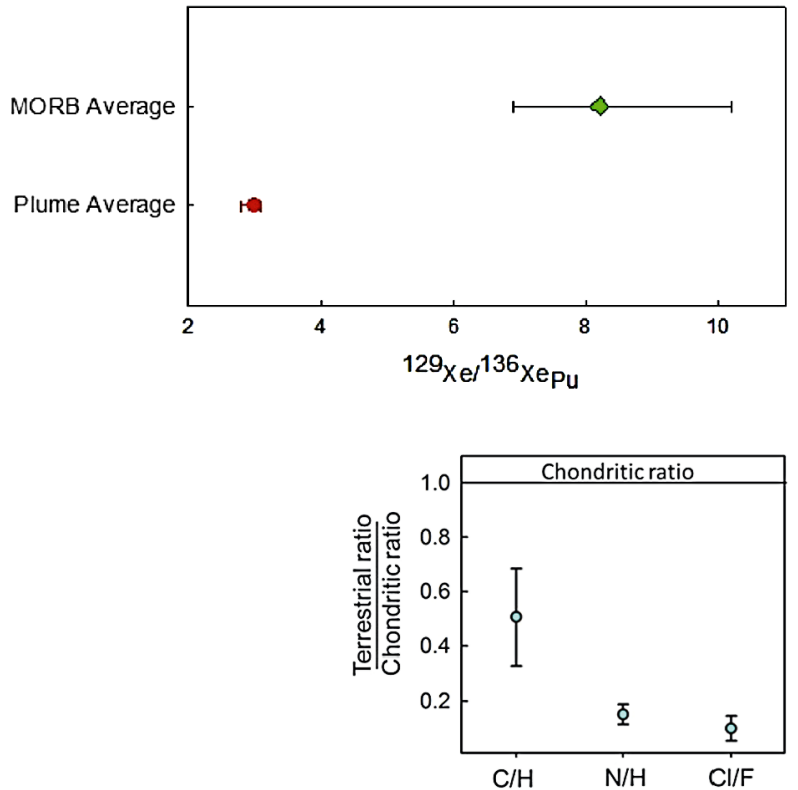

The relative abundances of the volatile elements also provide key insights into when volatiles must have been delivered. For example, the $\mathrm{C} / \mathrm{H}$ ratio and $\mathrm{N} / \mathrm{H}$ ratio on Earth is low compared to chondrites (Fig. 3), suggesting some process(es) fractionated this ratio following delivery of chondritic volatiles. For example, while the $\mathrm{N}$ and $\mathrm{H}$ isotopic ratios on Earth are chondritic, the N/H ratio does not match any known solar system object. Therefore, delivering all of the $\mathrm{H}$ and $\mathrm{N}$ associated with late accretion presents a significant challenge. Therefore Halliday (2013) concluded that water must have been delivered during the main phase of accretion. Halliday (2013) pointed out that if the entire $\mathrm{N}$ budget was delivered during the late accretion, the low $\mathrm{N} / \mathrm{H}$ ratio of the Earth has to imply that about $70 \%$ of the Earth's hydrogen, in the form of water, must have been delivered prior to the Moon forming impact. Tucker and Mukhopadhyay (2014) argued that the low $\mathrm{C} / \mathrm{H}$ and $\mathrm{N} / \mathrm{H}$ ratio reflects modification of the chondritic relative abundances of these volatile elements on the Earth. Likewise, even though the $\mathrm{Cl}$ isotopic composition of Earth is chondritic (Sharp and Draper 2013), the extremely low $\mathrm{Cl} / \mathrm{F}$ ratio of the Earth is an indication that the $\mathrm{Cl}$ abundance must have been modified relative to the $\mathrm{F}$ abundance (Tucker and Mukhopadhyay 2014). Hirschmann (2016) argued that while most of the water was delivered prior to the Moon forming giant impact, since the C/S ratio of the Earth is chondritic, and because both these elements are expected to have strongly partitioned into the Earth's core during core formation, significant $\mathrm{C}$ and $\mathrm{S}$ replenishment must have happened after cessation of core formation. That is, $\mathrm{C}$ and $\mathrm{S}$ replenishment of the Earth's mantle and exosphere must have occurred after the formation of the Moon in association with late accretion.

Hirschmann's (2016) argument about volatile delivery after the formation of the Moon is also compatible with the noble gases. The mantle and atmosphere have distinct nonradiogenic $\mathrm{Ne}, \mathrm{Kr}$ and importantly, while the atmosphere is enriched in the heavier ${ }^{22} \mathrm{Ne}$ isotope relative to the mantle, it is depleted in the heavier ${ }^{84} \mathrm{Kr}$ isotope compared to the mantle (Holland et al. 2009; Marty 2012; Halliday 2013). Hence, the mantle and atmosphere cannot be related through outgassing or outgassing combined with hydrodynamic escape. The simplest explanation for the difference in mantle and atmospheric composition would be for the 
majority of atmospheric noble gases to be delivered after the last major mantle-atmosphere exchange - the Moon forming giant impact. Delivery of the noble gases associated with the late accretion, however presents a challenge. The atmospheric Kr inventory requires that the late accretion was CI chondrites, constituting 4.5\% of Earth's mass (Marty 2012), a number that would lead to significant overabundances in the terrestrial budget of the platinum group elements. To resolve this, delivery of a small amount of cometary ices have been invoked (Halliday 2013; Marty et al. 2016). Laboratory experiments (Bar-Nun et al. 2007; Notesco et al. 2003) and recent measurements of comet 67P/Churyumov-Gerasimenko (Altwegg et al. 2015; Marty et al. 2016) suggest that cometary ices can trap significant amounts of noble gases. If the laboratory experiments and cometary measurements are representative of ices, the budget of atmospheric noble gases could be delivered by icy planetesimals representing $\sim 1 \%$ by mass of the late accretion. While the $\mathrm{H}$ and $\mathrm{N}$ isotopic compositions of comets can be significantly heavier than chondritic sources (e.g. Bockelée-Morvan et al. 1998; Meier et al. 1998; Jehin et al. 2009; Manfroid et al. 2009; Meech et al. 2009), accretion of such a small amount of cometary ice would lead to no detectable change in the $\mathrm{H}$ or $\mathrm{N}$ budget of the Earth. Therefore, the isotopic composition of these elements would not be perturbed away from chondritic values.

The compositions of the volatile elements therefore, suggest a complicated story of inheritance, loss and multiple stages of replenishment. Importantly, the budget of the different volatile elements appear to have grown at different rates, governed by the balance between delivery and loss, with loss to the core or outer space controlled by geochemical behavior of the elements. Acquisition of volatile elements started in the embryo stage, evidence of which survives in the $\mathrm{Ne}$, and possibly $\mathrm{H}$, isotopic compositions in the deep mantle plume source. With the dissipation of the nebula, planetary growth enters the giant impact phase of accretion-a stage marked by both addition of major volatiles with chondritic composition and volatile loss from the growing Earth. During this stage, water, and most likely all of the volatile elements, were partially retained. Following, the Moon forming giant impact, C, $\mathrm{N}, \mathrm{S}$, and halogens were partially replenished through accretion of chondritic planetesimals during the late accretion phase; the atmospheric noble gases where likely acquired from the accretion of $1 \times 10^{22} \mathrm{~g}$ of icy planetesimals, which is approximately $0.1 \%$ of the late accretion mass (Marty et al. 2016). This amount of icy material is not large enough to leave its mark on the abundance of the major volatiles $(\mathrm{H}, \mathrm{C}, \mathrm{S}, \mathrm{N}$, halogens). The delivery of the icy planetesimals could have occurred during main phase of the late accretion or at the very tail end of late accretion (e.g. Marty et al. 2016).

\subsection{What Were the Volatile Loss Processes and When Were Volatiles Lost?}

\subsubsection{Loss via Hydrodynamic Escape Versus Loss via Impacts}

As discussed in Sect. 2.1, there is evidence in the deep mantle that nebular gases were acquired but signatures of nebular gases are not seen in the shallower mantle or in the atmosphere. This observation suggests loss of volatiles from the Earth during the main accretion phase. A simple calculation can be done based on the radiogenic Xe budget to show significant volatile loss during accretion. The abundance of ${ }^{127} \mathrm{I}$ in the bulk silicate Earth is 5-10 ppb (Lodders and Fegley 1998; Avice and Marty 2014). With an initial ${ }^{129} \mathrm{I} /{ }^{127} \mathrm{I}$ ratio of $1.0 \times 10^{-4}$ (Ozima and Podosek 2002), the decay of ${ }^{129} \mathrm{I}$ to ${ }^{129} \mathrm{Xe}$ should have produced $\sim 1.5-3 \times 10^{13}$ moles of radiogenic ${ }^{129} \mathrm{Xe}$ on the Earth. The atmospheric abundance of radiogenic ${ }^{129} \mathrm{Xe}$ is $\sim 2.8 \times 10^{11}$ moles (Pepin 1991). The mantle budget is uncertain but is lower than the Xe budget of the atmosphere (Pepin and Porcelli 2002). Thus, $>97 \%$ of the 
radiogenic ${ }^{129} \mathrm{Xe}$ has been lost from the Earth. The recent discovery that xenon isotopes in the Archean atmosphere were less fractionated than modern air suggests xenon loss during the Hadean and Archean associated with a mass fractionating process (e.g. Pujol et al. 2011). However, even after accounting for this mass fractionating loss, $\sim 75 \%$ of the ${ }^{129} \mathrm{Xe}$ must have been lost prior to $\sim 60 \mathrm{Ma}$, the approximate age of the Moon-forming giant impact (Avice and Marty 2014). Hence, substantial xenon loss must have occurred during the giant impact phase of accretion. The significant loss of radiogenic ${ }^{129} \mathrm{Xe}$ during the giant impact phase of accretion should not be taken to imply that all of the major volatiles were delivered after the Moon-forming giant impact. Rather, the volatile abundances could be residual to loss followed by replenishment during the late accretion phase.

Hydrodynamic escape has been invoked to explain the loss of volatiles, including Xe (Pepin 1991; Pepin and Porcelli 2006; Dauphas 2003). Hydrodynamic loss, however, requires a H-rich atmosphere, such as would be present if a nebular atmosphere was captured by the proto-Earth. In fact, models for hydrodynamic escape start off with a solar composition gas and explain the present abundances through loss of the $\mathrm{H}_{2}$-rich atmosphere followed by resupply from the mantle and delivery of planetesimals during the late accretion (Pepin 1991; Dauphas 2003).

The present day geochemistry of volatiles, however, shows no evidence of hydrodynamic escape. Hydrodynamic escape fractionates the isotopes as the escaping $\mathrm{H}_{2}$ preferentially drags the lighter isotope, leaving the atmosphere enriched in the heavier isotopes. Therefore, the present day chondritic isotopic composition of the $\mathrm{H}, \mathrm{C}, \mathrm{N}$ and $\mathrm{Cl}$ would require the $\mathrm{H}_{2}$ flux to space to fall off at just the right time so that volatile escape is shut off exactly when chondritic isotopic composition are reached. Importantly, because the mass differences between the isotopes are different for the different elements, escape for the different volatile elements has to stop at different times and precisely at the point when the isotopic compositions have reached chondritic ratios. Such a scenario requires an extreme fine tuning of the $\mathrm{H}_{2}$ escape flux and appears highly implausible. Alternatively, to generate the present day isotopic compositions requires adding isotopically light volatiles sequestered in the mantle to the isotopically heavy residual atmosphere that was mass fractionated through hydrodynamic escape (Pepin 1991; Pepin and Porcelli 2002). However, recent measurements of $\mathrm{Kr}$ isotopes indicate that the mantle is actually enriched in the heavier isotopes compared to the atmosphere (Holland et al. 2009). Therefore, hydrodynamic escape is not the primary loss signature recorded in the present day volatile budget. The lack of evidence for hydrodynamic escape in the present day volatile signatures does not imply that hydrodynamic escape never occurred. Hydrodynamic escape might have taken place on embryos shortly after the nebular gas dissipated (e.g. Erkaev et al. 2014). For our present day planet, it appears that if hydrodynamic escape occurred early in the accretion history (embryo stage), the resulting chemical signature has been completely overprinted by volatile resupply and additional loss mechanisms that did not fractionate the isotopes.

The process capable of driving loss without isotopic fractionation is impacts, both giant impacts as well as impacts of planetesimals. Atmospheric erosion associated with planetesimals has recently been shown to be quite efficient (Schlichting et al. 2015). While impact-driven loss does not fractionate the isotopes since it involves bulk ejection of the atmosphere, impact-driven losses can fractionate the elements. This is because during impacts, water oceans and crust would be retained preferentially compared to the atmosphere. Thus, elements residing in the atmosphere (nitrogen, noble gases) would be lost in preference to those in the ocean and crust ( $\mathrm{H}$ and $\mathrm{C}$ ). In the rest of Sect. 2.2, we will discuss constraints on magma ocean outgassing and atmospheric loss via impacts from $\mathrm{He} / \mathrm{Ne}$ ratios in the mantle. 
Fig. $4{ }^{3} \mathrm{He} /{ }^{22} \mathrm{Ne}$ ratios in modern terrestrial reservoirs along with the ${ }^{3} \mathrm{He} /{ }^{22} \mathrm{Ne}$ ratios in possible sources that may have contributed primordial $\mathrm{He}$ and $\mathrm{Ne}$ to the Earth. Figure after Tucker and Mukhopadhyay (2014), with solar nebula value update from Heber et al. (2012)

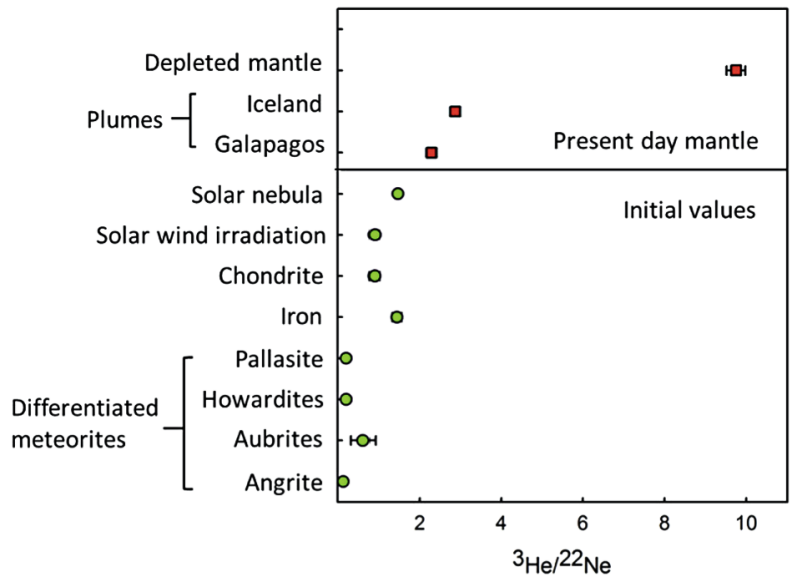

\subsubsection{The He/Ne Ratio Difference Between MORB and Plume Sources}

The ${ }^{3} \mathrm{He} /{ }^{22} \mathrm{Ne}$ ratio of the present day mantle spans a large range from $\leq 2$, and possibly down to 1 , to $\geq 10$ (Fig. 4, Raquin and Moreira (2009), Coltice et al. (2011), Tucker and Mukhopadhyay (2014), Péron et al. (2016)). Plumes with the most primitive ${ }^{21} \mathrm{Ne} /{ }^{22} \mathrm{Ne}$ ratios from Galapagos and Iceland have ${ }^{3} \mathrm{He} /{ }^{22} \mathrm{Ne}$ ratios of 2 to 3 (Kurz et al. 2009; Mukhopadhyay 2012; Raquin and Moreira 2009) while the depleted MORB mantle has a ${ }^{3} \mathrm{He} /{ }^{22} \mathrm{Ne}$ ratio $\geq 10$ with intermediate ${ }^{3} \mathrm{He} /{ }^{22} \mathrm{Ne}$ values due to mixing between the depleted mantle and the plume source (Tucker and Mukhopadhyay 2014). The ${ }^{3} \mathrm{He} /{ }^{22} \mathrm{Ne}$ ratio of the primitive reservoir sampled by plumes is higher than the nebular value by approximately a factor of $\sim 1$ to 2, with the depleted MORB source a factor of 6-10 higher than the sources of He and Ne to the Earth's mantle (Fig. 4).

Differences in the ${ }^{3} \mathrm{He} /{ }^{22} \mathrm{Ne}$ ratio between basalts from mid ocean ridges and basalts from mantle plumes have long been recognized (e.g. Honda and McDougall 1998; Moreira et al. 1998; Sarda et al. 2000). However, these differences were not universally accepted as reflecting a difference in the mantle sources of plumes and MORBs. Rather, magma generation and melt migration were sometimes invoked to explain the observed differences in ${ }^{3} \mathrm{He} /{ }^{22} \mathrm{Ne}$ ratios between MORBs and plumes (e.g. Sarda et al. 2000) with the mantle sources of basalts assumed to have similar ${ }^{3} \mathrm{He} /{ }^{22} \mathrm{Ne}$ ratios (e.g. Trieloff and Kunz 2005; Hopp and Trieloff 2008). More recent work however has unequivocally established that the mantle sources of plumes and MORBs have distinct ${ }^{3} \mathrm{He} /{ }^{22} \mathrm{Ne}$ ratios (Raquin and Moreira 2009; Kurz et al. 2009; Füri et al. 2010; Coltice et al. 2011; Marty 2012; Tucker and Mukhopadhyay 2014). Here we focus on the new interpretations of the ${ }^{3} \mathrm{He} /{ }^{22} \mathrm{Ne}$ ratio of mantle-derived basalts over the past few years.

\subsubsection{Magma Oceans and ${ }^{3} \mathrm{He} /{ }^{22} \mathrm{Ne}$ Ratios}

A striking feature of the ${ }^{3} \mathrm{He} /{ }^{22} \mathrm{Ne}$ ratios is that the mantle reservoirs have a higher value than even the Sun (Fig. 4). How did the Earth's interior acquire a ${ }^{3} \mathrm{He} /{ }^{22} \mathrm{Ne}$ ratio higher than even the solar nebula? To posit that the Earth's interior is enriched in ${ }^{3} \mathrm{He}$ compared to the Sun is unreasonable. More likely, the mantle must have preferentially lost ${ }^{22} \mathrm{Ne}$. Based on the factor of two higher solubility of $\mathrm{He}$ compared to $\mathrm{Ne}$ in a basaltic liquid, Honda and McDougall (1998) argued that magma ocean outgassing must have increased the MORB 
source ${ }^{3} \mathrm{He} /{ }^{22} \mathrm{Ne}$ ratio, as magma ocean degassing would preferentially release $\mathrm{Ne}$ over $\mathrm{He}$ into the atmosphere. They also noted that the lower ${ }^{3} \mathrm{He} /{ }^{22} \mathrm{Ne}$ of plumes might indicate a less degassed source that was largely isolated from the MORB source for most of Earth's history.

More recently, Coltice et al. (2011) suggested that the ${ }^{3} \mathrm{He} /{ }^{22} \mathrm{Ne}$ ratio in plumes reflect the signature of a basal magma ocean. In the aftermath of the last giant impact, the Moonforming impact, a global magma ocean may have existed. For a peridotite liquid, the liquidus intersects the isentrope in the mid-lower mantle (e.g. Mosenfelder et al. 2009; Thomas et al. 2012). Consequently, the magma ocean will first start crystallizing at the mid-lower mantle, which would ultimately isolate a layer of molten mantle above the core-mantle boundarythe basal magma ocean (BMO) (Labrosse et al. 2007). As crystallization in the BMO proceeds, incompatible elements, like noble gases, get enriched in the liquid. If both $\mathrm{He}-\mathrm{Ne}$ partition coefficients are on order 0.01 , the late crystallizing minerals could have relative high concentrations and low ${ }^{3} \mathrm{He} /{ }^{22} \mathrm{Ne}$ ratios. Since iron also becomes enriched in the residual liquid as the BMO crystallizes, the late crystallizing minerals would be Fe-enriched and likely to be more dense than the surrounding mantle assemblage. As a result of its highdensity, the low ${ }^{3} \mathrm{He} /{ }^{22} \mathrm{Ne}$ reservoir would be entrained to low degrees in the convective flow, preserving the reservoir over the age of the Earth. Coltice et al. (2011) noted that the factor of 5-10 higher value in the MORB is unexplained and could reflect mantle degassing, although they did not indicate when or how this degassing occurs.

Tucker and Mukhopadhyay (2014) argued that mantle outgassing associated with the long-term plate tectonic cycling is not likely to increase the ${ }^{3} \mathrm{He} /{ }^{22} \mathrm{Ne}$ ratio of the mantle. Specifically, the mechanisms they investigated for raising ${ }^{3} \mathrm{He} /{ }^{22} \mathrm{Ne}$ ratios were (i) partial melting that generates oceanic crust and a depleted mantle residue and (ii) recycling and mixing of oceanic crust and lithosphere back into the mantle. Given current determinations of partition coefficients for He and Ne (Brooker et al. 2003; Heber et al. 2007; Jackson et al. 2013), they found that partial melting is not an efficient process for fractionating He from $\mathrm{Ne}$ in the mantle. Likewise, due to ubiquitous incorporation of atmospheric noble gases, both upper and lower oceanic crust have $\mathrm{a}^{3} \mathrm{He} /{ }^{22} \mathrm{Ne}$ of $\sim 0$ (Staudacher and Allègre 1988; Moreira et al. 2003). If the recycling efficiency of He and $\mathrm{Ne}$ back into the mantle is high, recycling of oceanic crust should decrease the ${ }^{3} \mathrm{He} /{ }^{22} \mathrm{Ne}$ ratio and not increase it. Therefore, Tucker and Mukhopadhyay (2014) concluded ${ }^{3} \mathrm{He} /{ }^{22} \mathrm{Ne}$ values of 10 for the depleted mantle could not be due to processes associated with the long-term plate tectonic cycle. They suggested that outgassing or ingassing of a magma ocean is a process that is expected to raise the mantle ${ }^{3} \mathrm{He} /{ }^{22} \mathrm{Ne}$ ratio. As $\mathrm{He}$ is more soluble in magmas than $\mathrm{Ne}$, magma ocean ingassing or outgassing will raise the mantle ${ }^{3} \mathrm{He} /{ }^{22} \mathrm{Ne}$ ratios. The differences between the ${ }^{3} \mathrm{He} /{ }^{22} \mathrm{Ne}$ ratios of plume and depleted mantle sources then reflects a difference in deep versus shallow mantle magma ocean outgassing history of the Earth.

\subsubsection{Multiple Magma Oceans, Giant Impacts and Atmospheric Losses}

To explain the low ${ }^{3} \mathrm{He} /{ }^{22} \mathrm{Ne}$ ratios and solar-like ${ }^{20} \mathrm{Ne} /{ }^{22} \mathrm{Ne}$ ratios of $\geq 12.9$ in plumes, Tucker and Mukhopadhyay (2014) proposed ingassing of nebular gas from a gravitationally accreted nebular atmosphere into a magma ocean, a hypothesis that was previously proposed as the mechanism responsible for acquisition of terrestrial He and $\mathrm{Ne}$ (Harper and Jacobsen 1996; Mizuno et al. 1980; Porcelli et al. 2001; Yokochi and Marty 2004). During nebular ingassing into a magma ocean, the ${ }^{3} \mathrm{He} /{ }^{22} \mathrm{Ne}$ ratio of the magma ocean would be fractionated from the nebular value of $\sim 1.5$ by the $\mathrm{He} / \mathrm{Ne}$ solubility ratio of $\sim 2$ to values of $\sim 2.3-3$. The magma ocean ingassing occurs on an embryo, probably about the size of Mars or slightly larger. 


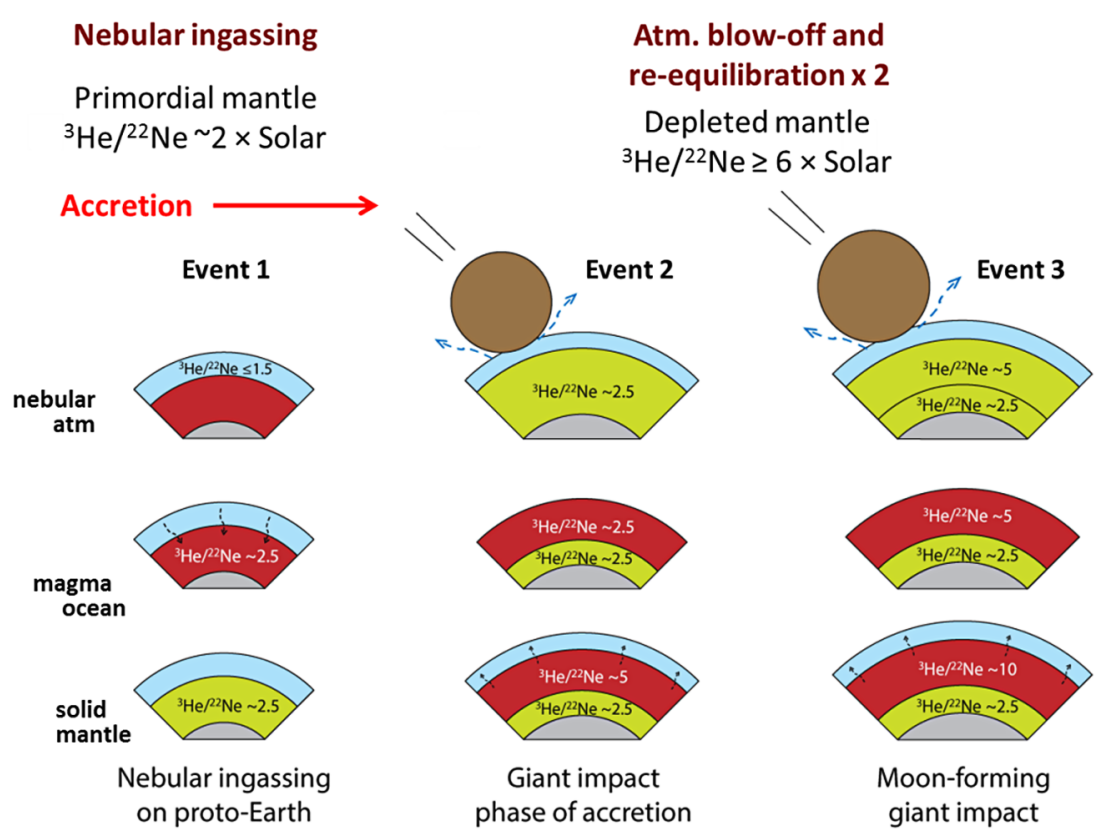

Fig. 5 A proposed chronology of events to explain the highly fractionated ${ }^{3} \mathrm{He} /{ }^{22} \mathrm{Ne}$ ratio of the depleted mantle. Figure after Tucker and Mukhopadhyay (2014)

The magma ocean on embryos will crystallize on a timescale on order $10^{6}$ to $10^{7}$ years (Zahnle et al. 2007; Elkins-Tanton 2008; Lebrun et al. 2013). The median lifetime of nebular gas is $\sim 2.5$ Myrs and the maximum observed timescale is $\sim 10 \mathrm{Myr}$ (e.g. Hillenbrand 2008). Consequently, the nebular gas will dissipate on a timescale similar to the crystallization timescale of the magma ocean. The gas in the disk has a dampening effect on planetary orbit. Consequently, dissipation of the nebular gas pumps up the eccentricity of the embryos, causing them to run into each other-the giant impact phase of terrestrial planet accretion. During the giant impact phase, magma oceans are expected as the proto-Earth grows to its present mass through violent collisions with other embryos.

Tucker and Mukhopadhyay (2014) proposed that after loss of the nebular atmosphere, and during the giant impact phase of terrestrial growth, at least two separate partial mantle magma ocean episodes raised the ${ }^{3} \mathrm{He} /{ }^{22} \mathrm{Ne}$ ratio of the shallower mantle from $\sim 2$ to $\geq 10$, with the last magma ocean associated with the Moon-forming giant impact (Fig. 5). However, during this phase, the low ${ }^{3} \mathrm{He} /{ }^{22} \mathrm{Ne}$ ratio in the deep mantle must be preserved. Thus, Tucker and Mukhopadhyay (2014) argued that the later giant impacts including the Moon forming giant impact may not have produced a whole mantle magma ocean because the timescale for turnover of a turbulently convecting magma ocean may be a short as a few weeks (Solomatov 2000; Elkins-Tanton 2008; Pahlevan and Stevenson 2007), and hence, the magma ocean would be expected to be chemically homogeneous. Alternatively, if whole mantle magma oceans were produced, some process must have prevented mixing within the magma ocean. One possibility to prevent such mixing might be the presence of a dense basal magma ocean prior to the impact (Nakajima and Stevenson 2015).

The requirement of 2 giant impacts and magma ocean outgassing episodes comes from the $\mathrm{He} / \mathrm{Ne}$ solubility ratio of $\sim 2$ in a magma ocean (Tucker and Mukhopadhyay 2014). Thus, each stage of magma ocean outgassing can only raise the residual liquid by at most a 
factor of 2. The degree of outgassing of a magma ocean is, however, tied to the atmospheric boundary condition above the magma ocean. If an atmosphere that had equilibrated with a previous magma ocean remained intact during a giant impact, the new magma ocean would not outgas and fractionate ${ }^{3} \mathrm{He}$ from ${ }^{22} \mathrm{Ne}$ because it would already be in equilibrium with the overlying atmosphere. Therefore, loss of the pre-existing atmosphere is a requirement for driving fractionation of He-Ne during magma ocean outgassing (Fig. 5). The loss of the nebular atmosphere in the aftermath of magma ocean ingassing on the planetary embryo could have been achieved through hydrodynamic escape (e.g. Pepin 1991), giant impacts (Genda and Abe 2003, 2005; Schlichting et al. 2015), impacts of planetesimals (Schlichting et al. 2015), or a combination of the above factors. During the giant impact phase of accretion, outgassed atmospheres are expected, which are likely to be $\mathrm{H}^{2} \mathrm{O}-\mathrm{CO}^{2}$ rich and not $\mathrm{H}^{2}$-rich (e.g. Heber et al. 2012). Thus, loss of an outgassed atmosphere is not likely to be driven by hydrodynamic escape. Rather, escape is more easily ascribed to giant impacts (Genda and Abe 2003) and/or impacts of planetesimals (Schlichting et al. 2015).

Complete loss of the atmosphere would lead to the largest extent of fractionation, the $\mathrm{He} / \mathrm{Ne}$ solubility ratio of $\sim 2$, with partial loss suppressing the extent of fractionation. Tucker and Mukhopadhyay (2014) noted that any process that leads to preferential He loss from the magma ocean or atmosphere, such as kinetically controlled degassing or hydrodynamic escape of the atmosphere, would also suppress the extent of $\mathrm{He}-\mathrm{Ne}$ fractionation during degassing of the magma ocean. In addition, He/Ne fractionation associated with degassing would also be suppressed if during the giant impact phase, meteoritic $\mathrm{He}$ and $\mathrm{Ne}$ with ${ }^{3} \mathrm{He} /{ }^{22} \mathrm{Ne}$ of $\sim 0.9$ were delivered to the Earth's mantle. In such cases, more than the two episodes of giant impacts may be required to drive the mantle ${ }^{3} \mathrm{He} /{ }^{22} \mathrm{Ne}$ ratio to values of $\sim 10$. The proposed chronology of events in Fig. 5 should therefore, be viewed as a minimum sequence of events during the formation of the Earth.

In summary, the observation of different ${ }^{3} \mathrm{He} /{ }^{22} \mathrm{Ne}$ ratios in the plume and MORB mantle reservoirs requires the formation and preservation of two distinct mantle domains during accretion (Coltice et al. 2011; Tucker and Mukhopadhyay 2014). High ${ }^{3} \mathrm{He} /{ }^{22} \mathrm{Ne}$ ratio of $\geq 10$ for the MORB source suggests multiple magma oceans and multiple atmospheric loss episodes. The lower ${ }^{3} \mathrm{He} /{ }^{22} \mathrm{Ne}$ of the plume source also appears to be associated with a magma ocean; Coltice et al. (2011) suggested that the plume values are the signature of crystallization of a basal magma ocean while Tucker and Mukhopadhyay (2014) associated it within ingassing of nebular gases into a magma ocean on the proto Earth. Tucker and Mukhopadhyay (2014) argued that given the He/Ne solubility ratio of 2, the low ${ }^{3} \mathrm{He} /{ }^{22} \mathrm{Ne}$ ratios in plumes is not likely to have its origin in a basal magma ocean produced by the last giant impact (the Moon-forming giant impact) because that magma ocean must have produced the high ${ }^{3} \mathrm{He} /{ }^{22} \mathrm{Ne}$ ratio now sampled in the depleted mantle. However, a basal magma ocean sequestering low ${ }^{3} \mathrm{He} /{ }^{22} \mathrm{Ne}$ ratios in the deep mantle could have formed from a previous whole-mantle magma ocean episode and recent work suggests that a dense basal magma ocean layer may not get mixed in with the rest of the mantle in the aftermath of a giant impact (e.g. Nakajima and Stevenson 2015).

\subsubsection{Terrestrial Volatile Signatures and Atmospheric Loss}

Geochemical evidence for multiple magma oceans associated with multiple giant impacts is consistent with dynamic simulations of terrestrial planet formation that suggest more than just the Moon-forming giant impact (e.g. Chambers and Wetherill 1998; Chambers 2001). The atmospheric loss episodes put forth to explain the fractionation of $\mathrm{He} / \mathrm{Ne}$ during magma ocean outgassing, also provide explanations for other features of the Earth's volatile signatures, such as the depletion of carbon, and the very large depletion of nitrogen, with respect 
to water (Fig. 3). Carbon depletion has previously been linked to atmospheric loss or to partitioning of carbon to the core (Hirschmann and Dasgupta 2009; Hirschmann 2016). Likewise, $\mathrm{N}$ depletion has been linked to core formation (Marty 2012). However, since carbon appears to be more siderophile than nitrogen by factors of 25 to 100 , depletion of $\mathrm{N}$ by core formation is unlikely because it would be accompanied by a significantly greater depletion of $\mathrm{C}$ (Tucker and Mukhopadhyay 2014; Hirschmann 2016). The observed moderate depletion in $\mathrm{C}$ and extreme depletion in $\mathrm{N}$ might be explained due to atmospheric loss associated with impacts. Carbon can form bicarbonate and carbonate ions and as a result, a significantly larger fraction of $\mathrm{C}$ can be the ocean (or crust) compared to N. Since both giant impacts and impacts of planetesimals preferentially remove the atmosphere over an ocean (Genda and Abe 2005; Schlichting et al. 2015), atmospheric loss would lead to large fractionation in the $\mathrm{N} / \mathrm{H}$ ratio and a more modest fractionation in the $\mathrm{C} / \mathrm{H}$ ratio (Fig. 3). Since impacts lead to bulk ejection of the atmosphere, the $\mathrm{C}$ and $\mathrm{N}$ isotopic composition of the Earth would remain chondritic.

Atmospheric loss may also explain why chlorine is depleted in the Earth relative to fluorine (Fig. 3). Compared to $\mathrm{Cl}, \mathrm{F}$ is more soluble in magmas by a factor of 4 (Andrews et al. 2009). Consequently, magma ocean degassing would lead to preferential transport of $\mathrm{Cl}$ to the Earth's surface compared to $\mathrm{F}$. If chlorine is lost due to giant impacts or impacts of planetesimals, it would explain the depletion of $\mathrm{Cl}$ with respect to $\mathrm{F}$. On the Earth's surface, chlorine would be expected to reside primarily in the ocean and crust unless a steam atmosphere was present. Thus, loss of chlorine might also require loss of water, either in the form of partial ocean loss or through erosion of a steam atmosphere. Overall, Earth's volatile budget records the violent events during accretion. While late accretion contributed volatiles to Earth (e.g. Albarède 2009; Marty 2012; Hirschmann 2016), late accretion did not overprint the volatile characteristics acquired during the main stages of Earth's accretion (Halliday 2013; Tucker and Mukhopadhyay 2014; Dauphas and Morbidelli 2014).

\section{Atmospheric Loss by Small and Large Impacts}

Terrestrial planet formation consists of successive mergers of small planetesimals and larger protoplanets accumulating into final planets ranging in size from Mars to Earth. The delivery of volatiles and atmospheric erosion are intricately linked to planetary growth since planetesimal impacts and giant impacts can both deliver volatiles and lead to their loss due to atmospheric erosion.

We present here simple analytic models which we use to calculated the atmospheric mass loss due to impacts during planet formation. The aim of these models is to distill the somewhat complex and complicated nature of impacts to their essential physics, which allows us to gain an intuitive understanding of atmospheric mass loss results and to apply them over the whole range of possible impactor sizes. These models are not suitable to determine the precise outcome of a single specific impact, which for example have been investigated by Shuvalov (2009) and Liu et al. (2015), but capture the overall mass loss results in an averaged sense, e.g. once averaged over various impact geometries and angles.

For the purpose of this chapter we assume that the atmosphere is, to first order, isothermal such that its density profile is exponential and given by

$$
\rho=\rho_{0} \exp [-z / h]
$$

where $\rho_{0}$ is the density on the ground, $z$ the height in the atmosphere measured from the ground and $h$ the atmospheric scale height. At the end of this chapter we will discuss how 
Fig. 6 Illustration of the impact geometry. Planetesimal impacts can only eject atmosphere locally. Treating their impact as a point-like explosion leading to an isotropic shock at the impact site, the maximum atmospheric mass that they can eject in a single impact is given by all the mass above the tangent plane, which is a fraction given by $h / 2 R$ of the total atmospheric mass. Figure after Schlichting et al. (2015)
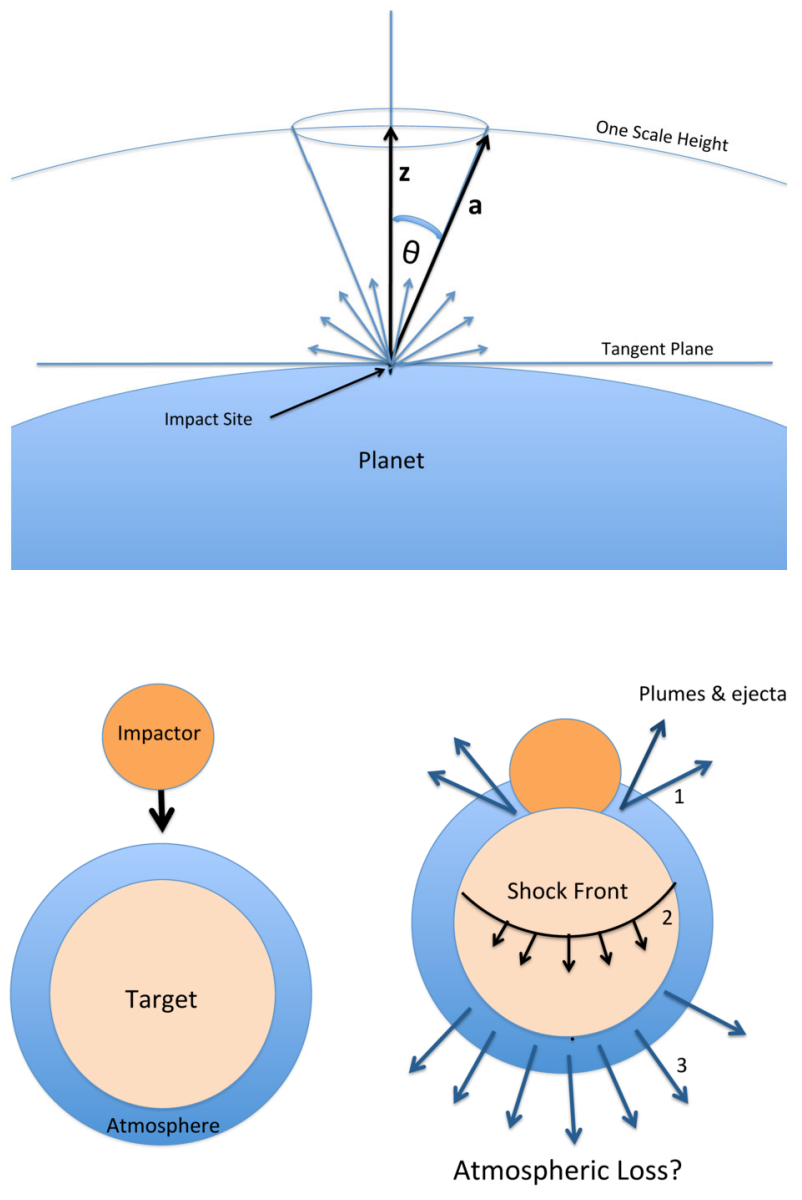

Fig. 7 Illustration of a giant impact. 1) The giant impact ejects atmosphere and ejecta close to the impact point and launches a strong shock. 2) The shock front propagates through the target causing a global ground motion. 3) This ground motion in turn launches a strong shock into the planetary atmosphere, which can lead to loss of a significant fraction of or even the entire atmosphere. Figure after Genda and Abe (2003) and Schlichting et al. (2015)

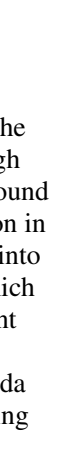

the results derived here will change for an adiabatic atmosphere in which the heat transport is facilitated by convection. We assume here that the atmosphere is planar, which is valid for the terrestrial planets since their atmospheric scale heights, $h$, are small compared to their radii, $R$. For the current Earth, $h / R \sim 0.1 \%$ so the planar condition is indeed well justified for the terrestrial planets. In contrast, for close in exoplanets the planar condition breaks down and full spherical treatment should be used, see for example Inamdar and Schlichting (2015, 2016).

Impacts can lead to atmospheric losses in two distinct ways: First, the expansion of plumes generated at the impact site can erode the atmosphere locally, but not globally (see Fig. 6). Such loss can be caused by small planetesimal impacts and is discussed in detail immediately below. Second, when the target and impactor are comparable in mass, a giant impact can create a strong shock that transverses through the entire planet leading to global ground motions that in turn can launch a shock into the atmosphere (see Fig. 7). The shock accelerates the atmosphere, and fluid parcels that are accelerated to above the escape velocity of the target are lost. A simple model calculating the atmospheric mass loss due to giant impacts is described in Sect. 3.3. 


\subsection{Atmospheric Loss by Small Impacts}

Small impacts can individually only eject planetary atmospheres locally, close to their impact sites. However, collectively they play an important role in atmospheric loss during planet formation, since they are many orders of magnitude more efficient per unit impactor mass in atmospheric erosion than giant impacts (Schlichting et al. 2015).

\subsubsection{Mass Loss Regimes}

The first calculations of atmospheric loss by planetesimal impacts used the Zel'dovich and Raizer (1967) solution for the expansion of a vapour plume and compared the momentum of the expanding gas and the mass of the overlying atmosphere (e.g. Melosh and Vickery 1989; Vickery and Melosh 1990; Ahrens 1993). Here we use a similar approach and compare our analytic results to atmospheric mass loss results from numerical simulations. When the Earth or a protoplanet is hit by a small impactor, the impactor's velocity is rapidly decelerated and its kinetic energy is converted into heat and pressure leading to something analogous to an explosion. We model the impact as an isotropic point-like explosion on the surface of the target. Assuming momentum conservation and impact velocities comparable to the escape velocity, $v_{e s c}$, yields an ejecta mass comparable to the impactor mass, $m_{\text {Imp }}$, that propagates isotropically with a velocity of order $v_{\text {esc }}$ into a half-sphere centered on the impact site. This implies that atmosphere is only lost where its mass per unit solid angle, $m_{\theta}$, as measured from the impact site, is less than that of the ejecta, which is equal to $m_{\text {Imp }} / 2 \pi$.

More generally we find for an arbitrary impact velocity and momentum transfer efficiency in the impact, $\eta$, that atmosphere is only ejected within a solid angle, $\theta$, where

$$
m_{\theta} \leq \eta\left(\frac{v_{\text {Imp }}}{v_{\text {esc }}}\right) \frac{m_{\text {Imp }}}{2 \pi} .
$$

For the density profile given in (2) we have

$$
m_{\theta}=2 \pi \rho_{0} \int_{0}^{\infty} \exp \left[-\left(a^{2}+2 a R \cos \theta\right) / 2 R h\right] a^{2} d a,
$$

where $z$ is the height in the atmosphere above the ground and it is related to $a$, the distance from the impact site to the top of the atmosphere (see Fig. 6), by $z=\left(a^{2}+2 a R \cos \theta\right) / 2 R$. Note, the integration in (4) is only over $a$ and not $\theta$ since the explosion at the impact site is assumed to be isotropic (Schlichting et al. 2015). This leads to two interesting limits:

1) There is a lower limit on the impactor size that can eject any atmosphere.

2) There is a lower limit on the impactor size that can eject all the atmospheric mass above the tangent plane.

The first limit can be derived by equating the mass residing in the vertical column above the impact site to (3). Integrating (4) for $\theta=0$ yields a minimum impactor mass of

$$
m_{\min }=4 \pi \rho_{0} h^{3} \eta^{-1}\left(\frac{v_{e s c}}{v_{\text {Imp }}}\right),
$$

which reduces to the result obtained by Schlichting et al. (2015) for $v_{\text {imp }} \sim v_{\text {esc }}$ and $\eta \sim 1$, and yields $r_{\min }=\left(3 \rho_{0} / \rho_{\text {imp }}\right)^{1 / 3} h \sim 1 \mathrm{~km}$ for the current Earth (Fig. 8a). 
The second limit is obtained by integrating (4) for $\theta=\pi / 2$ which yields the minimum impactor mass that can eject all the mass above the tangent plane, $m_{c a p}$, and is given by

$$
m_{\text {cap }}=\sqrt{2} \rho_{0}(\pi h R)^{3 / 2} \eta^{-1}\left(\frac{v_{\text {esc }}}{v_{\text {Imp }}}\right),
$$

which again reduces to the result obtained by Schlichting et al. (2015) for $v_{\text {Imp }} \sim v_{\text {esc }}$ and $\eta \sim 1$, and yields $r_{\text {cap }}=\left(\rho_{0} / \rho_{\text {imp }}\right)^{1 / 3}(h R)^{1 / 2} \sim 25 \mathrm{~km}$ for the current Earth (Fig. 8c). All impactors above this size will eject all the atmospheric mass above the tangent plane but not more than that, unless they are so large that they are in the giant impact regime which is discussed in Sect. 3.3.

\subsubsection{Mass Loss Efficiencies}

Next we turn to calculating the mass loss efficiencies for the various impactor sizes. The mass loss efficiency is given by the amount of atmospheric mass lost divided by the impactor mass needed to achieve the loss. These two quantities are not equal because we model the explosion at the impact site as isotropic whereas the atmospheric mass follows an isothermal or adiabatic density profile (see (2)). As a result the atmospheric mass directly vertically above the impact site is easiest to eject, since it has the lowest column density and the atmospheric mass along the tangent plane is hardest to eject since it has the largest column density. The atmospheric mass residing inside a cone subtended by an angle $\theta$ measured from the normal to the impact site (see Fig. 8) is

$$
\mathcal{M}_{\text {Eject }, \theta}=2 \pi \rho_{0} \int_{a=0}^{a=\infty} \int_{\theta^{\prime}=0}^{\theta^{\prime}=\theta} \exp [-z / h] \sin \theta^{\prime} a^{2} d \theta^{\prime} d a .
$$

The mass loss efficiency is given by dividing (7) by (4) and evaluating it for various impactor sizes. Figure 9 shows the mass loss efficiency as a function of impactor size. Small impactors with $r_{*}=\sqrt{3} r_{\text {min }}$ are the most efficient impactors per unit mass in ejecting the atmosphere. For the current Earth this corresponds to bodies with $r \sim 2 \mathrm{~km}$. The value of $\mathcal{M}_{\text {Eject }} / m_{\text {Imp }}$ decreases rapidly for larger planetesimals because the atmosphere along the tangent plane is harder to eject, due to its larger column density. In addition, once impactors are larger than $r_{c a p}$, each impactor only ejects the mass residing above the tangent plane, which reduces the mass loss efficiency per unit impactor mass.

Integrating over the whole cap, i.e. from $\theta=0$ to $\theta=\pi / 2$, yields a total cap mass of

$$
\mathcal{M}_{\text {cap }}=2 \pi \rho_{0} h^{2} R,
$$

in the limit that $R \gg h$, which applies for the terrestrial planets. This is the maximum atmospheric mass that a single planetesimal impact can eject and is given by all the mass above the tangent plane of the impact site. The ratio of the mass in the cap compared to the total atmospheric mass is therefore $\mathcal{M}_{\text {cap }} / M_{\text {atmos }}=h / 2 R$.

\subsubsection{Impactor Size Distribution}

Figure 9 shows the ratio of atmospheric mass ejected to impactor mass. To understand the atmospheric mass loss over time we calculate the atmospheric loss due to a population of planetesimal impactors of various sizes. The impactors are assumed to follow a power-law size distribution such that the cumulative impactor flux can be parametrized as $N(>r)=$ 
Fig. 8 Different planetesimal impact regimes. The size of the shaded hemisphere is scaled to the impactor mass (assuming an impact velocity comparable to the escape velocity) such that the mass per unit solid angle in the hemisphere presented by the shaded region is given by $m_{\text {Imp }} / 2 \pi$ as measured radially outward from the impact site. Panel a) illustrates the limit in which the impactor is able to only eject the atmosphere vertically above the impact site, which corresponds to $m_{\min }=4 \pi \rho_{0} h^{3}$. Panel b) corresponds to an intermediate regime, where the impactor is able to eject all the atmospheric mass residing in the red cone. Panel c) displays the limit in which an impactor is large enough to eject all the atmospheric mass residing above the tangent plane of the impact site, which corresponds to a minimum impactor mass of $m_{\text {cap }}=\sqrt{2} \rho_{0}(\pi h R)^{3 / 2}$ a)

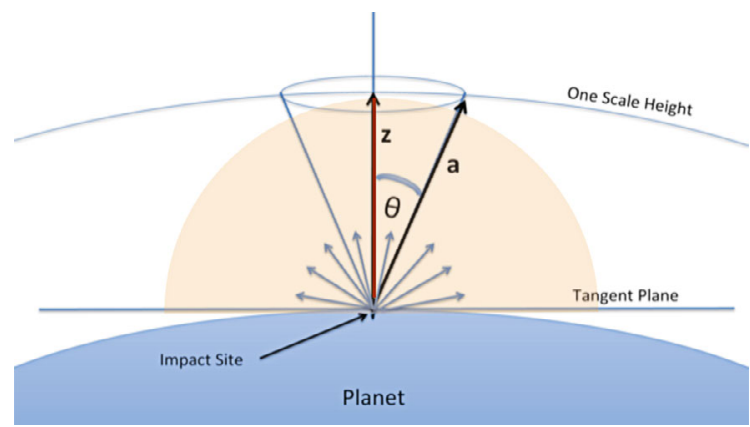

b)

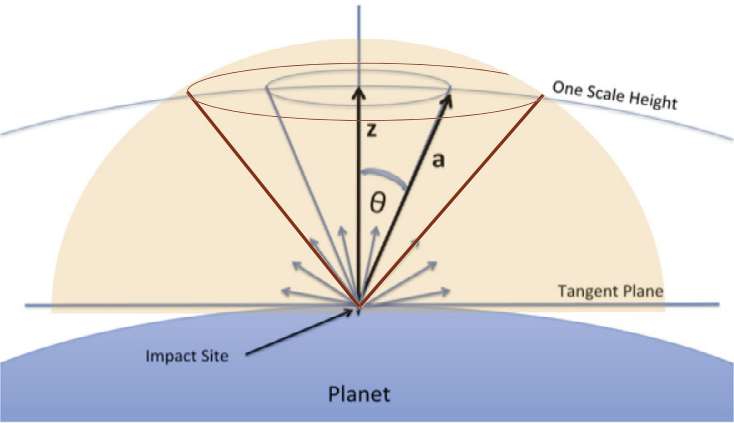

c)

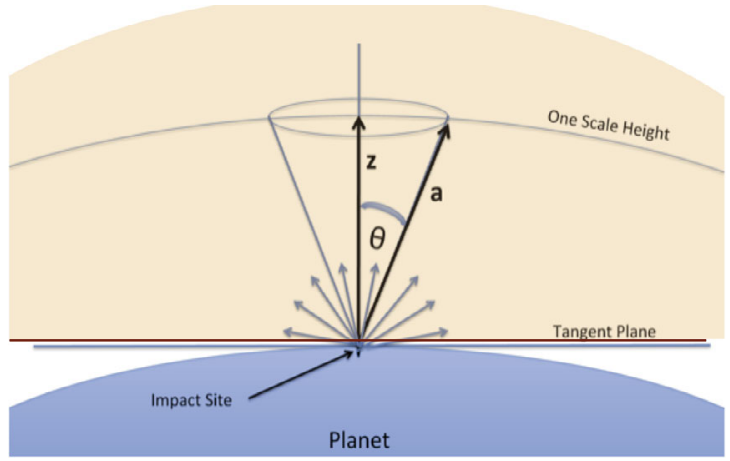

$N_{0}\left(r / r_{0}\right)^{-q+1}$, where $q$ is the differential power law index and $N_{0}$ is normalized to impactors of size $r_{0}$ and is the impactor flux (number per unit time per unit area). The atmospheric mass loss rate is then given by

$$
\frac{d M_{\text {atmos }}}{d t}=-\pi R^{2} \frac{N_{0}(q-1)}{r_{0}} \int_{r_{\min }}^{r_{\max }}\left(\frac{r}{r_{0}}\right)^{-q} \mathcal{M}_{\text {Eject }}(r) d r,
$$




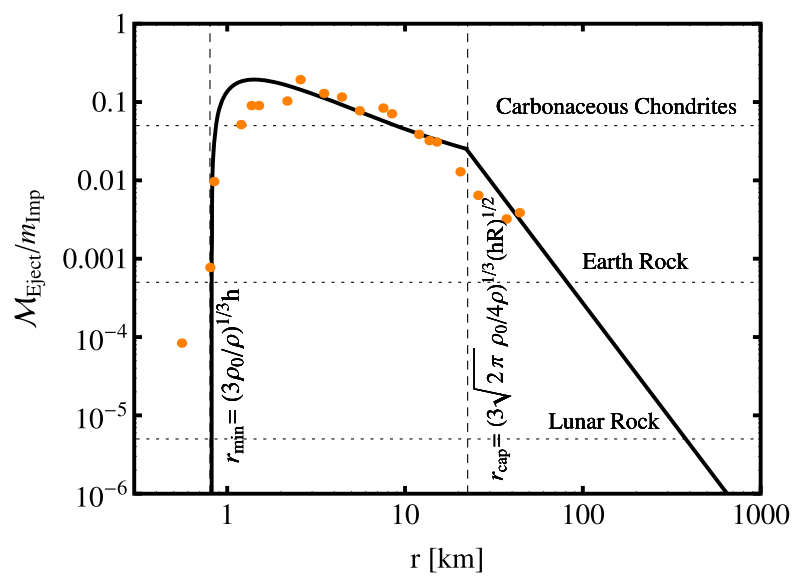

Fig. 9 Ratio of atmospheric mass ejected to impactor mass, $\mathcal{M}_{\text {Eject }} / m_{\text {Imp }}$. Numerical values are scaled to the current Earth's atmosphere and shown for $v_{\text {imp }} \eta / v_{\text {esc }} \sim 1$. Small impactors with $r_{*}=\sqrt{3} r_{\text {min }}$ are the most efficient impactors per unit mass in ejecting the atmosphere and the ejection efficiency decreases rapidly for larger planetesimals. Whether or not planetesimal impacts will lead to a net loss of planetary atmospheres depends on the impactor sizes distribution, their volatile budget and the amount of outgassing their impacts can initiate. The three dotted horizontal lines correspond to volatile contents of $5 \mathrm{wt} \%$ (representative of some of the most water rich carbonaceous chondrites), $0.05 \mathrm{wt} \%$ (representative of the average water content in the bulk Earth excluding the hydrosphere) and $0.0005 \mathrm{wt} . \%$ corresponding to an estimate of the minimum water content of the bulk moon (McCubbin et al. 2010). For comparison, data from oblique impact simulations for escape velocities of $11.2 \mathrm{~km} / \mathrm{s}$ and impact velocities of $30 \mathrm{~km} / \mathrm{s}$ from Shuvalov (2009) are shown by the orange points. Figure after Schlichting et al. (2015)

where $\mathcal{M}_{\text {Eject }}(r)$ is the atmospheric mass ejected due to an impactor with radius $r$. Solving (9) yields

$$
M_{\text {atmos }}(t)=M_{0}\left(1-\frac{t}{t^{*}}\right)^{3 /(q-1)},
$$

where $M_{0}$ is the initial atmospheric mass at $t=0$ and $t_{*}$ is the time it takes to erode the entire atmosphere (Melosh and Vickery 1989). Equation (9) shows that the whole atmosphere is eroded in a finite time, which is due to the fact that as the atmosphere is lost, its density decreases and even smaller impactors, which are more numerous, start to contribute to the mass loss. For $1<q<3$, the value for $t^{*}$ is

$$
t_{q<3}^{*}=\frac{6}{\pi(q-1) C R h N_{0}}\left(\sqrt{\frac{\pi h}{8 R}} \frac{M_{0}}{m_{0}}\right)^{(q-1) / 3},
$$

and for $q>3$ it is

$$
t_{q>3}^{*}=\frac{3(q-3)}{\pi(q-1) h^{2} N_{0}}\left(\left(\frac{h}{R}\right)^{2} \frac{M_{0}}{m_{0}}\right)^{(q-1) / 3},
$$

where $m_{0}=4 \pi \rho_{\text {imp }} r_{0}^{3} / 3$ (Schlichting et al. 2015). We get two different expressions for $t^{*}$ depending on the power-law index of the planetesimal size distribution because for $1<q<3$ atmospheric mass loss is dominated by impactors that remove the whole atmospheric mass above the tangent plane and for $q>3$ the atmospheric mass loss is dominated by impactors that each only eject part of the atmospheric mass residing above the tangent plane. 


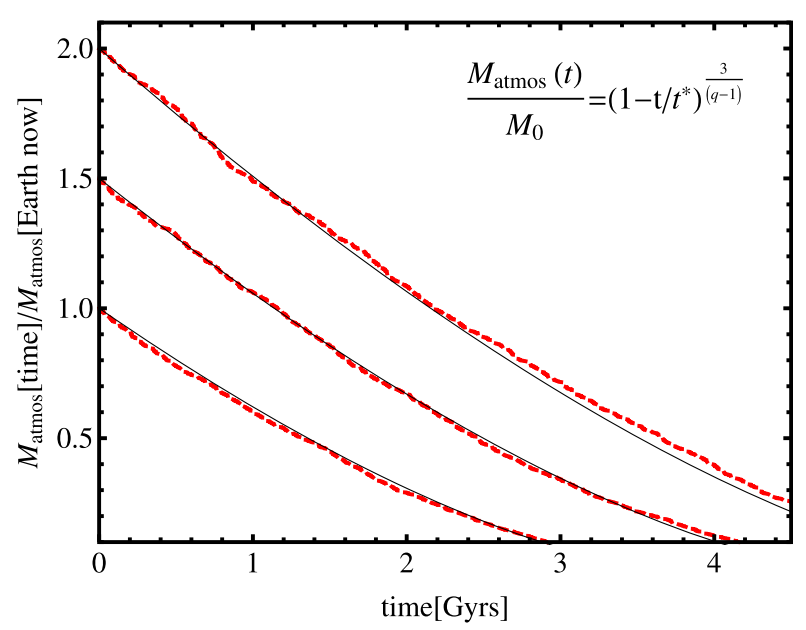

Fig. 10 Atmospheric mass loss over time for three different initial atmospheric masses normalized to the current Earth's atmosphere. The impactor population contains a total mass of $0.1 \%$ Earth's masses, consistent with the mass inferred for the late veneer, and has a power-law size distribution with a differential power-law index of $q=2.8$ as inferred from the lunar cratering record by Neukum et al. (2001). The largest impactor is $300 \mathrm{~km}$ in size and the smallest $1 \mathrm{~m}$. The impactor flux is assumed to be uniform over 4.5 Gyrs, but the total atmospheric mass loss would remain the same for a varying impactor flux over time as long as the total impactor mass remains unchanged. When calculating the mass loss efficiency we assumed $v_{\text {imp }} \eta / v_{\text {esc }} \sim 1$. The thick-red dashed lines are from numerical Monte Carlo simulations that calculate the atmospheric loss due to each individual impact and the thin black lines are the analytic solutions given in (10)

The constant $C$ in (11) parameterizes any additional contribution to the atmospheric loss by impactors that are smaller than those that eject all the mass above the tangent planet. Melosh and Vickery (1989) investigated the mass loss due to impactors that remove all the atmosphere above the tangent plane (i.e. the $1<q<3$ regime) but they assumed that $\mathcal{M}_{\text {cap }}=m_{\text {cap }}$ (instead of $\mathcal{M}_{\text {cap }}=m_{\text {cap }}(2 h / \pi R)^{1 / 2}$ see (6) and (8)), ignoring the fact that the atmospheric column density is larger in the direction along the tangent plane than directly vertically above the impact site, and they also neglected the numerical coefficient $C$ discussed above.

Figure 10 shows atmospheric erosion over time due to an impactor size distribution with $q=2.8$ and an impactor flux that bombards the Earth uniformly over 4.5 Gyrs with a total of $0.1 \%$ of the mass of the Earth. The largest body is $300 \mathrm{~km}$ and the smallest $1 \mathrm{~m}$ in radius. The thin black lines correspond to the analytic solution given in (10) and the red dotted lines to numerical simulations that calculate the atmospheric loss after each impact and determine the evolution of the atmospheric mass over time. As expected, the numerical and analytical results are in excellent agreement. They demonstrate that small impactors that contain about $0.1 \%$ of the mass of the Earth, which is about the mass inferred for the late veneer, can erode the entire Earth's atmosphere, if the Earth's initial atmospheric mass was similar to its current one (Schlichting et al. 2015). Whether such a bombardment by small impactors leads to a net loss or gain in atmospheric mass depends on the volatile content of the impactors themselves, how much of their volatiles end up in the atmosphere and the amount of volatiles released from the impact melt pools that are created by the impacts. 


\subsection{Atmospheric Loss by Giant Impacts}

Giant impacts are believed to be the last major assembly stage of terrestrial planet formation (e.g. Chambers 2001) and may also play a major role in the formation of close-in exoplanets that have masses between that of Earth and Neptune (e.g. Raymond et al. 2008; Hansen and Murray 2012; Inamdar and Schlichting 2015). The atmospheric survival during a giant impact has been examined by several groups both in the context of terrestrial planet formation (Genda and Abe 2003, 2005; Stewart et al. 2014; Schlichting et al. 2015) and in that of close-in exoplanets (Inamdar and Schlichting 2015, 2016). These works integrate the hydrodynamic equations of motion of the planetary atmosphere and determine the amount of atmospheric mass loss for a given ground velocity that launches a strong shock into the atmosphere. Recently Liu et al. (2015) presented the first results of three dimensional hydrodynamic simulations of atmospheric mass loss that model the giant impact as well as the atmospheric mass loss together. Here we calculate the atmospheric loss due to a given ground motion. We assume that the atmosphere is planar. In addition we assume that radiative cooling can be neglected such that the flow is adiabatic. The hydrodynamic equations describing the flow are then given by

$$
\begin{aligned}
\frac{1}{\rho} \frac{D \rho}{D t}+\frac{\partial u}{\partial z} & =0, \\
\frac{D u}{D t}+\frac{1}{\rho} \frac{\partial p}{\partial z} & =0, \\
\frac{1}{p} \frac{D p}{D t}-\frac{\gamma}{\rho} \frac{D \rho}{D t} & =0,
\end{aligned}
$$

where $\gamma$ is the adiabatic index and $D / D t$ the ordinary Stokes time derivative. The solutions to the hydrodynamic equations above can be separated into their time-dependent and spatial parts allowing for analytic self-similar solutions (Raizer 1964; Grover and Hardy 1966; Schlichting et al. 2015). Specifically we have,

$$
\begin{aligned}
& \rho(z, t)=\rho_{0} \exp [-Z(t) / h] G(\zeta), \quad u(z, t)=\dot{Z} U(\zeta), \\
& p(z, t)=\rho_{0} \exp [-Z(t) / h] \dot{Z}^{2} P(\zeta)
\end{aligned}
$$

where $Z(t)$ is the position of the shock front and $\zeta=(z-Z(t)) / h$. The similarity variables for the density, velocity and pressure are given by $G(\zeta), U(\zeta)$ and $P(\zeta)$, respectively. Using the expressions in (16) and substituting them into the hydrodynamic equations (13)-(15) yields for the spatial parts

$$
\begin{aligned}
\frac{1}{G} \frac{d G}{d \zeta}(U-1)+\frac{d U}{d \zeta} & =1 \\
(U-1) \frac{d U}{d \zeta}+\frac{1}{G} \frac{d P}{d \zeta} & =-\frac{U}{\alpha} \\
(U-1)\left(\frac{1}{P} \frac{d P}{d \zeta}-\frac{\gamma}{G} \frac{d G}{d \zeta}\right) & =-\frac{2}{\alpha}-\gamma+1,
\end{aligned}
$$

and a time dependent part given by

$$
\frac{\dot{Z}^{2}}{\ddot{Z}}=\alpha h \text {. }
$$


Fig. 11 Mass loss fraction, $\chi_{\text {loss }}$, as a function of $v_{g} / v_{\text {esc }}$ for an adiabatic atmosphere in black and an isothermal atmosphere shown as red-dashed line. Both loss curves correspond to and adiabatic index of $\gamma=5 / 3$
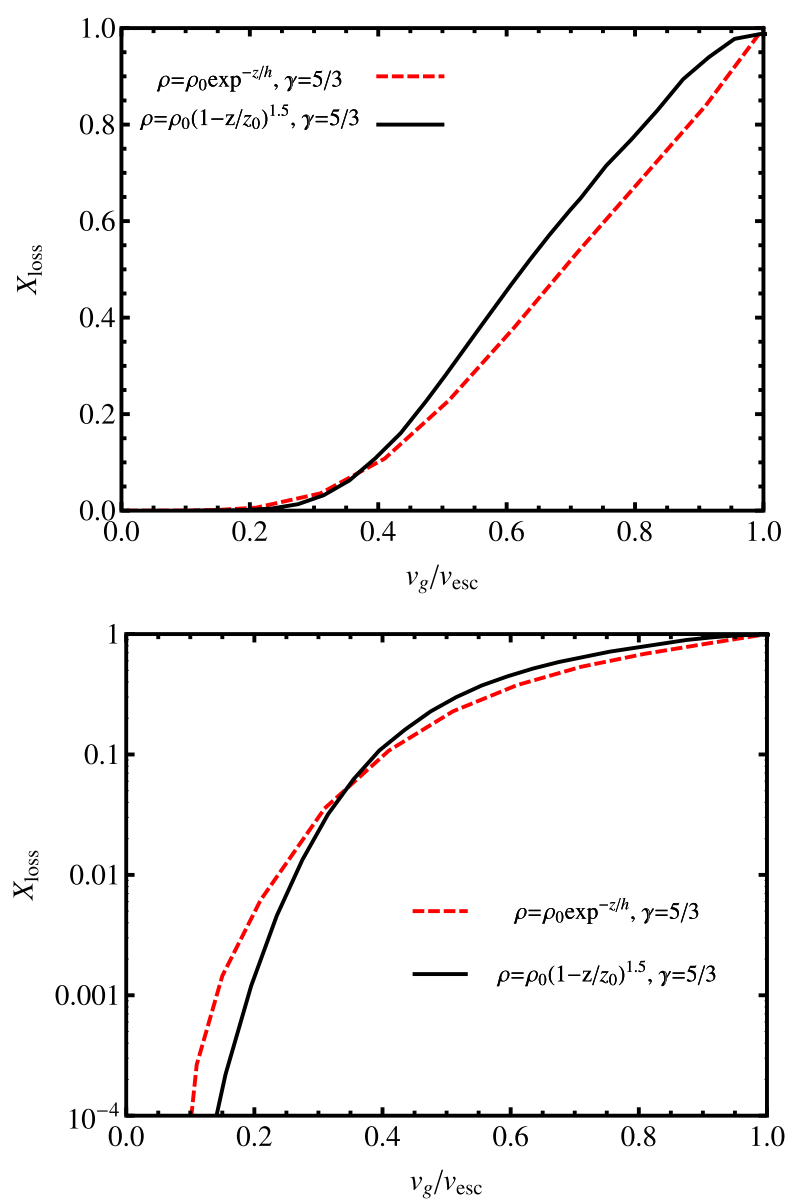

Using the strong shock conditions we have

$$
G(0)=\frac{\gamma+1}{\gamma-1}, \quad U(0)=\frac{2}{\gamma+1}, \quad P(0)=\frac{2}{\gamma+1} .
$$

We refer the reader to Schlichting et al. (2015) for a full derivation of the self-similar solutions to Equations (17)-(20). Figure 11 shows the atmospheric mass loss fraction as a function of the ground velocity with which the shock is launched into the atmosphere, where the atmospheric mass loss fraction is defined as

$$
\chi_{\text {loss }}=\exp \left[-z_{\text {esc }} / h\right]
$$

where $z_{\text {esc }}$ is the initial height in the atmosphere of the fluid element that has been accelerated to a velocity equal to the escape velocity by the shock, such that the atmosphere at $z \geq z_{\text {esc }}$ will be lost. 
Fig. 12 Illustration of the impact geometry. An impactor of mass, $m$, and impact velocity, $v_{i m p}$, impacts a target with mass, $M$, and radius, $R$. Assuming momentum conservation, we calculate the shocked fluid velocity, $v_{s}$, and the component of the ground velocity normal to the surface, $v_{g}$, as a function of the distance from the impact point. Figure after Schlichting et al. (2015)

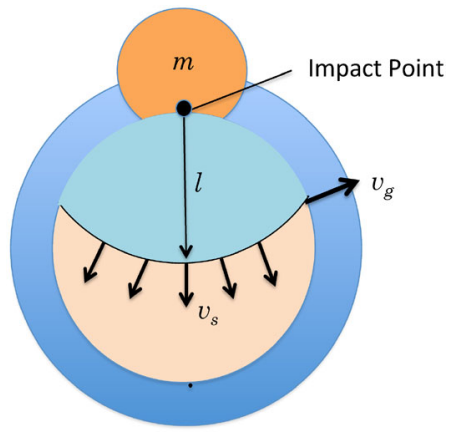

\subsubsection{Global Mass Loss}

Above we calculated the atmospheric mass loss due to a given ground velocity. In order to calculate the total global atmospheric mass loss, we need to relate the ground velocity to the impactor's mass and impact velocity. We do this by using a simple model following Schlichting et al. (2015). We approximate the impact as a point like explosion on the planet's surface, which results in a self-similar solution of the second type (Zel'dovich and Raizer 1967). As the shock propagates through the planet, its velocity must fall off faster than dictated by energy conservation but slower than required by momentum conservation. This is because as the shock propagates it must lose energy, but its momentum is increased by the nonzero pressure in the target. Catastrophic impact simulations find scaling laws that are close to ones resulting from momentum conservation (Love and Ahrens 1996; Benz and Asphaug 1999; Leinhardt and Stewart 2009). Assuming momentum conservation and a constant density target, the velocity of the shocked fluid traveling through the protoplanet is

$$
v_{s}=v_{\text {Imp }}\left(\frac{m}{M}\right) \frac{1}{(l / 2 R)^{3}(4-3(l / 2 R))},
$$

where $l$ is the distance of the shock travelled from the impact point, (see Fig. 12). The ground velocity with which the shock is launched into the atmosphere is due to the component of the shocked fluid velocity that is perpendicular to the planet's surface, such that $v_{g}=v_{s} l /(2 R)$, which yields

$$
v_{g}=v_{\text {Imp }}\left(\frac{m}{M}\right) \frac{1}{(l / 2 R)^{2}(4-3(l / 2 R))} .
$$

Using this ground velocity we can now calculate the global atmospheric mass loss by summing the loss from all the location on the planet for the various ground velocities. The resulting atmospheric loss as a function of $\left(v_{\text {Imp }} / v_{\text {esc }}\right)(m / M)$ for an isothermal atmosphere is shown in Fig. 13. The total atmospheric loss consists of two components: The first is from the area of the planet's surface where the ground motion is large enough such that locally all the atmosphere is lost (dashed line in Fig. 13), the second component corresponds to the region of the planet where the local ground velocity is small enough such that only part of the atmosphere is lost (thin solid line in Fig. 13) (Schlichting et al. 2015). Independent of the exact value of the adiabatic index, we find that the global atmospheric mass loss fraction is for an isothermal atmosphere well approximated by

$$
X_{\text {loss }}=0.4\left(\frac{v_{\text {Imp }} m}{v_{\text {esc }} M}\right)+1.4\left(\frac{v_{\text {Imp }} m}{v_{\text {esc }} M}\right)^{2}-0.8\left(\frac{v_{\text {Imp }} m}{v_{\text {esc }} M}\right)^{3}
$$


Fig. 13 Global mass loss fraction (thick solid line), calculated by taking into account the different ground velocities across the planet's surface. The total atmospheric loss consists of two components: the first is from the area of the planet's surface where the ground motion is large enough such that locally all the atmosphere is lost (dashed line) and the second component corresponds to the regions of the planet's surface where the local ground velocity is too small to lead to complete loss but large enough such that a fluid parcel residing higher in the atmosphere is accelerated to velocities exceeding the escape velocity such that the upper parts of the atmosphere are lost (thin solid line). The analytic fit given by the equation in the top panel is shown as dotted line. Figure after Schlichting et al. (2015)
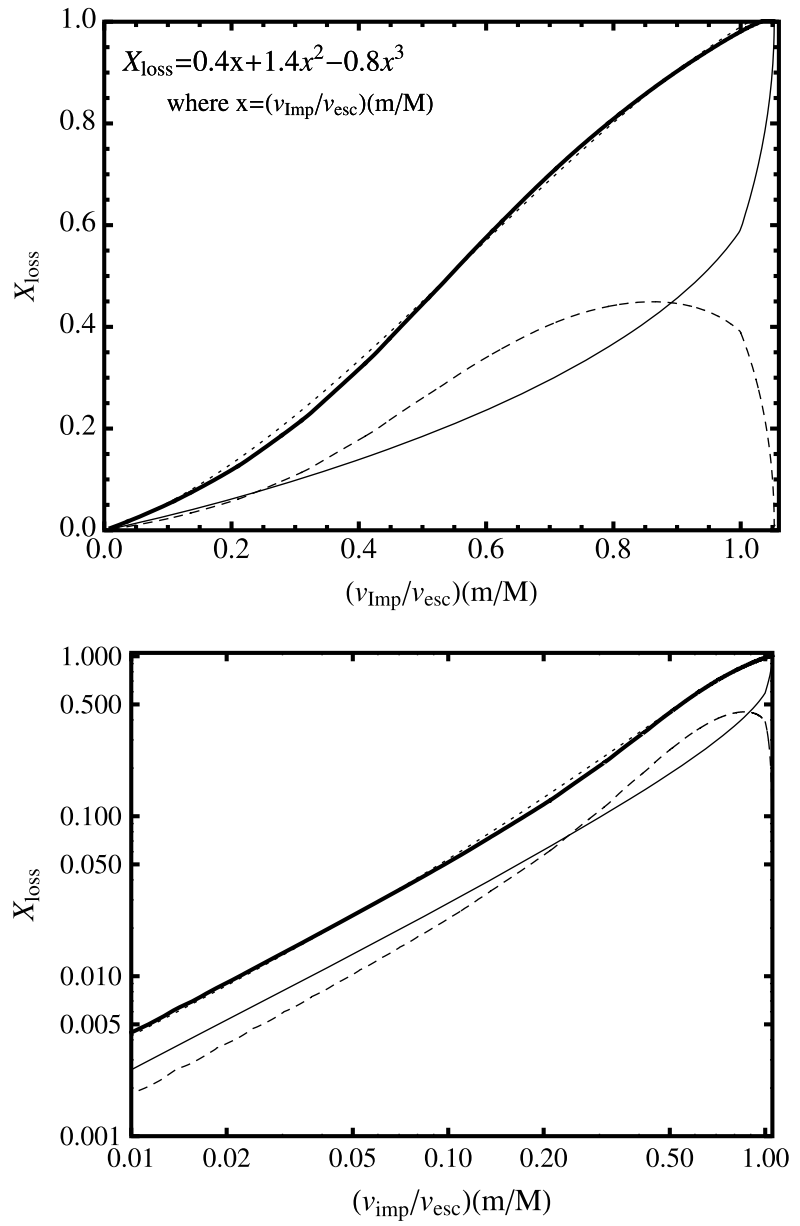

whereas for an adiabatic atmosphere we find

$$
X_{\text {loss }}=0.4\left(\frac{v_{I m p} m}{v_{\text {esc }} M}\right)+1.8\left(\frac{v_{\text {Imp }} m}{v_{\text {esc }} M}\right)^{2}-1.2\left(\frac{v_{\text {Imp }} m}{v_{\text {esc }} M}\right)^{3} .
$$

In both cases, $X_{\text {loss }} \simeq 0.4(m / M)\left(v_{\text {Imp }} / v_{\text {esc }}\right)$ in the limit that $\left(v_{\text {Imp }} / v_{\text {esc }}\right)(m / M) \ll 1$. We find a mass loss fraction of $6 \%$ for an Mars-sized impactor hitting an $0.9 M_{\oplus}$ protoplanet with $v_{\text {imp }} \sim v_{\text {esc }}$, which is about a factor of 2 lower than estimates by Genda and Abe (2003) who assumed a single averaged ground velocity of $4-5 \mathrm{~km} / \mathrm{s}$ for all locations on the planet. The presence of an ocean can significantly enhance the atmospheric mass loss (Genda and Abe 2005). Lock et al. (2014) find that with a large ocean up to $30 \%$ of the atmosphere can be lost in the canonical Moon-forming impact.

\subsection{Comparison of Atmospheric Loss Across All Impact Regimes}

We now turn to comparing the three different atmospheric mass loss regimes derived above. First we turn to the regime, where the mass loss is due to small impactors that can only 
eject a fraction of the atmospheric mass above the tangent plane, i.e. $r_{\min }<r<r_{\text {cap }}$. In this regime the total mass in impactors needed, assuming that all bodies have the same size, to remove the current Earth's atmosphere is (Schlichting et al. 2015)

$$
M_{T}=\frac{M_{\text {atmos }} m_{\text {Imp }}}{\mathcal{M}_{\text {Eject }}}=\frac{2 r}{r_{\text {min }}}\left(1-\left(\frac{r_{\text {min }}}{r}\right)^{2}\right)^{-1} M_{\text {atmos }} .
$$

This is strictly speaking an overestimate of the total impactor mass needed because as the atmospheric mass is decreasing with each impact, each new impactor is able to remove an ever larger fraction of the atmospheric mass above the tangent plane.

Next we turn to the second regime, in which impactors are large enough such they can eject all the atmosphere above the tangent plane, such that $r_{c a p}<r<r_{G I}$. In this regime, the total mass in impactors needed to erode the atmosphere is

$$
M_{T}=\frac{M_{\text {atmos }} m_{\text {Imp }}}{\mathcal{M}_{\text {Eject }}}=\frac{4 \pi}{3} \rho_{\text {imp }} r^{3} \frac{2 R}{h} .
$$

Since impactors with $r_{c a p}<r<r_{g i}$ are always limited to eroding all the atmospheric mass above the tangent plane, atmospheric mass loss does not become easier or more efficient over time as the atmospheric mass declines.

Finally we have giant impacts, which define the third regime. Following Schlichting et al. (2015), we define the transition from planetesimal impacts to giant impacts at an impactor radius at which a giant impact ejects more atmosphere than a smaller impactor that erodes all the mass above the tangent plane. This yields giant impacts for bodies with $r>r_{g i} \simeq$ $\left(2 h R^{2}\right)^{1 / 3}$. For the values of the current Earth $r_{g i} \simeq 900 \mathrm{~km}$. In the giant impact regime, the total mass in impactors needed to eject the atmosphere is

$$
M_{T}=\frac{M_{\text {atmos }} m_{\text {Imp }}}{\mathcal{M}_{\text {Eject }}} \simeq 4 M=\text { constant }
$$

where we assumed that $X_{\text {loss }} \ll 1$ in a single giant impact (see Schlichting et al. 2015 for details).

Figure 14 summarizes the three distinct impact regimes discussed above. It displays the total mass in impactors needed, i.e. $M_{T}=M_{\text {atmos }} / \mathcal{M}_{\text {Eject }}$, to lose the whole atmosphere as a function of impactor radius. Figure 14 impressively shows that, per unit impactor mass, small impactors with $r_{\text {min }}<r<r_{\text {cap }}$ are the most efficient in eroding the atmosphere. In fact for the current atmospheric mass of the Earth, they are more than five orders of magnitude more efficient (per unit impactor mass), than giant impacts. For Venus like atmospheres, they are still more than three orders of magnitude more efficient than giant impacts. The enormous atmospheric mass loss efficiency of small impactors is due to the fact that most of their impact energy and momentum is directly available for local mass loss, where as in the giant impact regime a lot of energy and momentum is 'wasted' by having to create a strong shock that transverses the entirety of the planet such that global atmospheric loss can be achieved. This explains intuitively why for giant impacts $M_{T} / M_{\oplus} \sim 1$ whereas for small planetesimal impacts $M_{T} / M_{\oplus} \sim M_{\text {atmos }}$.

\section{Summary and Conclusions}

Geochemical measurements and analytic impact models demonstrate that the formation of the terrestrial planets consisted of a rich interplay between volatile accretion and atmospheric mass loss facilitated by small and large impacts throughout the planet formation 


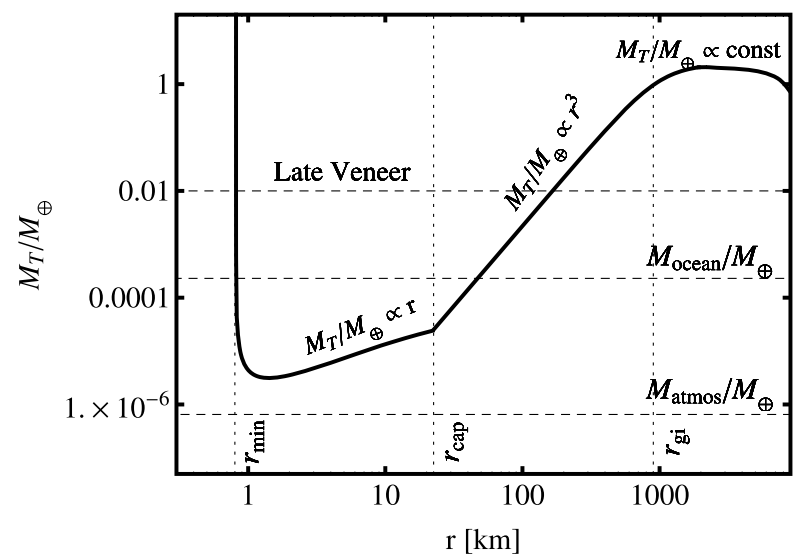

Fig. 14 Total impactor mass, $M_{T}$, needed to eject the atmosphere as a function of impactor radius, $r$. Three distinct ejection regimes are apparent: 1) For small $r_{\min }<r<r_{\text {cap }}$ (i.e., $1 \mathrm{~km} \lesssim r \lesssim 25 \mathrm{~km}$ ), the number of bodies needed scales roughly as $r^{-2}$. 2) For intermediate impactor sizes (i.e. $25 \mathrm{~km}<r<1000 \mathrm{~km}$ ), $N$ is constant, because each impact ejects the whole atmospheric cap, and to eject the entire atmosphere one needs $N=M_{\text {atoms }} / \mathcal{M}_{\text {cap }}=(2 R / h)$ number of impacts. 3) For larger impactor radii (i.e., $r>1000 \mathrm{~km}$ ) the impactors are large enough to initiate a shock wave traveling through the entire Earth and launching a shock into the atmosphere globally such that $N$ tends to 1 as $r$ tends to $R_{E a r t h}$. In the giant impact regime, $N \sim(R / r)^{3}$. Impactors with $r<r_{\text {min }} \sim 1 \mathrm{~km}$ are not able to eject any atmosphere. For comparison, the upper, middle, and lower dashed lines correspond to the mass ratio of the late veneer to the Earth's mass, the Earth's oceans to its total mass, and the Earth's atmosphere to its total mass, respectively. Figure after Schlichting et al. (2015)

process. A visual summary of this chapter and the accretion history of the terrestrial planets is given in Fig. 15. The following chronology of terrestrial planet formation and volatile accretion emerges: The solar nebula persisted for a few Myrs (Wang et al. 2017), during which dozens to hundred planetary embryos comparable to the size of Mars formed. Since these bodies formed in large part during the presence of the gas disk, they had the chance to accrete a primordial $\mathrm{H} / \mathrm{He}$ dominated atmosphere from the gas disk. Much of this primordial atmosphere was lost. Nebular $\mathrm{Ne}$ in the deep mantle might be a fingerprint of this early accretion, but most of the mantle does not remember this signature implying that volatile loss occurred during accretion likely due to a combination of collisions, photo-evaporation and hydrodynamic escape. Once the growing planetary embryos contain collectively more mass than the planetesimal population, their mutual gravitational excitation can no longer be efficiently damped by dynamical friction provided by the planetesimal population. As a result, the planetary embryos experience eccentricity growth, orbit crossing and giant impacts. Geochemical data indicate that most of the water could have been delivered prior to the Moon forming impact and that the Moon forming impact did not drive off the ocean. However, present day geochemistry of volatiles shows no evidence of hydrodynamic escape as the isotopic composition of most volatiles are chondritic. The relative abundances of the volatiles are however not in chondritic proportions, suggesting that impacts played a major role in sculpting volatile budgets. Geochemical evidence of atmospheric loss comes from the ${ }^{3} \mathrm{He} /{ }^{22} \mathrm{Ne}$, halogen ratios (e.g., $\mathrm{F} / \mathrm{Cl}$ ) and low $\mathrm{H} / \mathrm{N}$ ratios. Global magma oceans create an outgassed atmosphere during the giant impact phase. The outgassed atmospheres are eroded through a combination of large and small impacts, with smaller impacts being more efficient in eroding the atmosphere. Finally, during late accretion material is added to the Earth consisting of a mix of chondritic and icy planetesimals is added to the Earth. These impactors 

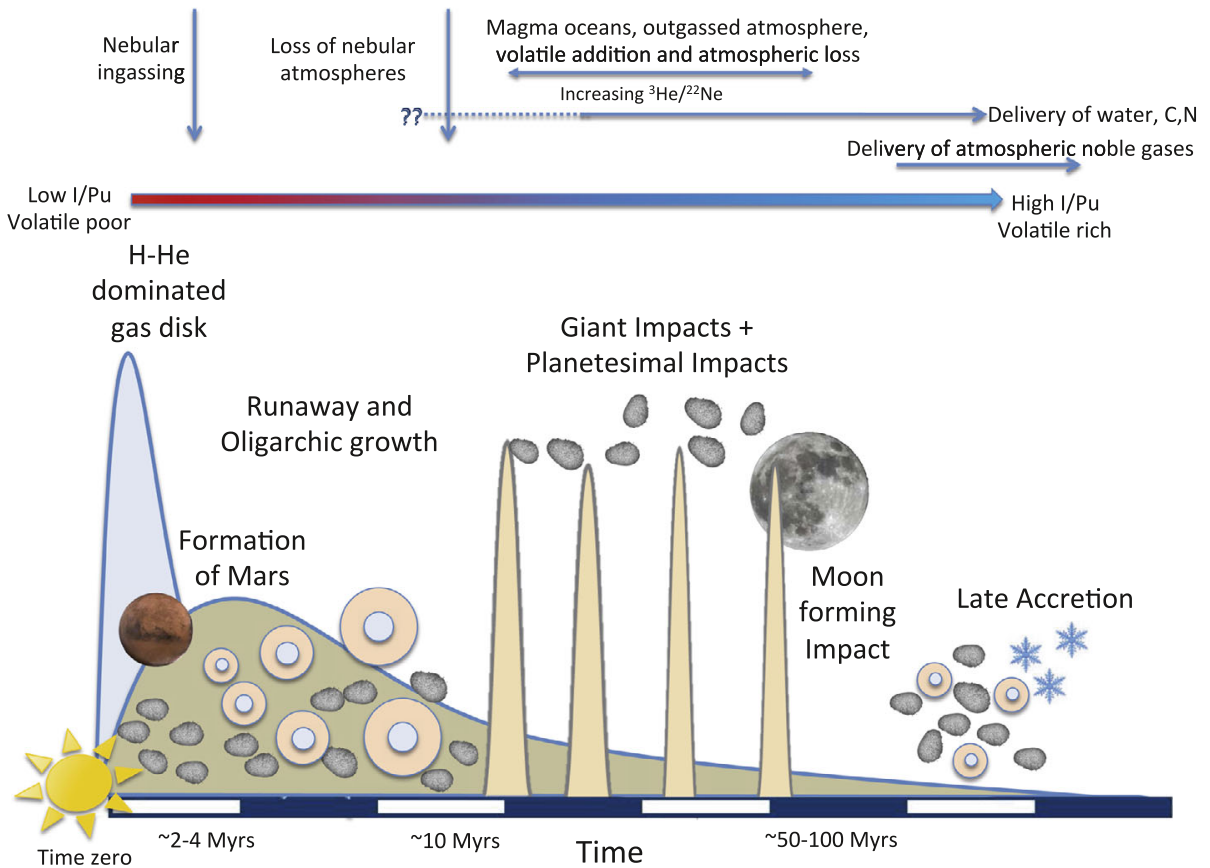

Fig. 15 Chronology of the terrestrial planets' accretion and volatile delivery. The solar system's gas disk (represented by the blue curve) dissipated after a few Myrs, which is comparable to the timescale for runaway growth resulting in the formation of Mars and dozens to hundred similar sized or larger planetary embryos (represented as differentiated cores). Planetary embryos continue to grow during oligarchic growth by accreting planetesimals (represented as grey boulders) and merging with comparable sized bodies. Some of these embryos form early enough to accrete a primordial $\mathrm{H} / \mathrm{He}$ dominated atmosphere from the gas disk, however much of this primordial atmosphere was likely lost due to collisions, photo-evaporation and hydrodynamic escape. Although nebular $\mathrm{Ne}$ and possible $\mathrm{H}$ in the deep mantle might be a fingerprint of this early accretion, most of the mantle does not remember this signature implying that volatile loss occurred during accretion. Once the growing planetary embryos contain collectively more mass than the planetesimal population, (the planetesimal population's decease in mass is shown by the brown curve), the mutual gravitational excitation of the planetary embryos can no longer be efficiently damped by dynamical friction provided by the planetesimal population. As a result, the planetary embryos undergo eccentricity growth, orbit crossing and giant impacts. Global magma oceans create an outgassed atmosphere during the giant impact phase. In addition, volatiles are delivered and atmospheres are eroded by planetesimal impacts and giant impacts. Geochemical ratios indicate that most of the water could have been delivered prior to the Moon forming impact and that the Moon forming impact did not drive off the ocean. Finally, during late accretion material is added to the Earth consisting of a mix of chondritic and icy planetesimals. These late impactors deliver volatiles, lead to outgasing and atmospheric loss. The interplay of these processes determined the atmospheric conditions of the early Earth

could have supplemented the existing volatile inventory. The icy planetesimals may have been particularly important for the delivery of the atmospheric noble gases as these were likely added after the Moon-forming impact. While these late impactors may have delivered volatiles, they could have also lead to outgasing and atmospheric loss. Whether impactors lead to a net loss or gain in atmospheric mass depends on the volatile content of the impactors themselves, how much of their volatiles end up in the atmosphere and the amount of volatiles released from the impact melt pools that are created by the impacts. Combining geochemical observations with impact models suggest an interesting synergy between small and big impacts, where giant impacts create large magma oceans and small impacts domi- 
nate the atmospheric loss. In fact, since small planetesimal impacts tend to be much more efficient (per unit impactor mass) in driving atmospheric loss than giant impacts, they likely dominated the atmospheric loss during terrestrial planet formation whereas giant impacts likely dominated the outgassing by creating large magma oceans. The early Earth's atmosphere was therefore likely determined by an interplay between volatile delivery, outgassing and atmospheric erosion.

Open Access This article is distributed under the terms of the Creative Commons Attribution 4.0 International License (http://creativecommons.org/licenses/by/4.0/), which permits unrestricted use, distribution, and reproduction in any medium, provided you give appropriate credit to the original author(s) and the source, provide a link to the Creative Commons license, and indicate if changes were made.

\section{References}

C.B. Agnor, R.M. Canup, H.F. Levison, On the character and consequences of large impacts in the late stage of terrestrial planet formation. Icarus 142, 219-237 (1999). https://doi.org/10.1006/icar.1999.6201

T.J. Ahrens, Impact erosion of terrestrial planetary atmospheres. Annu. Rev. Earth Planet. Sci. 21, 525-555 (1993). https://doi.org/10.1146/annurev.ea.21.050193.002521

F. Albarède, Volatile accretion history of the terrestrial planets and dynamic implications. Nature 461, $1227-$ 1233 (2009). https://doi.org/10.1038/nature08477

C.M.O.. Alexander, R. Bowden, M.L. Fogel, K.T. Howard, C.D.K. Herd, L.R. Nittler, The provenances of asteroids, and their contributions to the volatile inventories of the terrestrial planets. Science 337, 721 (2012). https://doi.org/10.1126/science.1223474

K. Altwegg, H. Balsiger, A. Bar-Nun, J.J. Berthelier, A. Bieler, P. Bochsler, C. Briois, U. Calmonte, M. Combi, J. De Keyser, P. Eberhardt, B. Fiethe, S. Fuselier, S. Gasc, T.I. Gombosi, K.C. Hansen, M. Hässig, A. Jäckel, E. Kopp, A. Korth, L. LeRoy, U. Mall, B. Marty, O. Mousis, E. Neefs, T. Owen, H. Rème, M. Rubin, T. Sémon, C.-Y. Tzou, H. Waite, P. Wurz, 67P/Churyumov-Gerasimenko, a Jupiter family comet with a high D/H ratio. Science 347(27), 1261952 (2015). https://doi.org/10.1126/ science. 1261952

S.M. Andrews, D.J. Wilner, A.M. Hughes, C. Qi, C.P. Dullemond, Protoplanetary disk structures in Ophiuchus. Astrophys. J. 700, 1502-1523 (2009). https://doi.org/10.1088/0004-637X/700/2/1502

G. Avice, B. Marty, The iodine-plutonium-xenon age of the Moon-Earth system revisited. Philos. Trans. R. Soc. Lond. Ser. A, Math. Phys. Sci. 372, 20130260 (2014). https://doi.org/10.1098/rsta. 2013.0260

C.J. Ballentine, B. Marty, B. Sherwood Lollar, M. Cassidy, Neon isotopes constrain convection and volatile origin in the Earth's mantle. Nature 433, 33-38 (2005). https://doi.org/10.1038/nature03182

A. Bar-Nun, G. Notesco, T. Owen, Trapping of $\mathrm{N}_{2}, \mathrm{CO}$ and $\mathrm{Ar}$ in amorphous ice: application to comets. Icarus 190, 655-659 (2007). https://doi.org/10.1016/j.icarus.2007.03.021

W. Benz, E. Asphaug, Catastrophic disruptions revisited. Icarus 142, 5-20 (1999). https://doi.org/10.1006/ icar.1999.6204

D. Bockelée-Morvan, D. Gautier, D.C. Lis, K. Young, J. Keene, T. Phillips, T. Owen, J. Crovisier, P.F. Goldsmith, E.A. Bergin, D. Despois, A. Wootten, Deuterated water in comet C/1996 B2 (Hyakutake) and its implications for the origin of comets. Icarus 133, 147-162 (1998). https://doi.org/10.1006/ icar.1998.5916

R.A. Brooker, Z. Du, J.D. Blundy, S.P. Kelley, N.L. Allan, B.J. Wood, E.M. Chamorro, J.-A. Wartho, J.A. Purton, The 'zero charge' partitioning behaviour of noble gases during mantle melting. Nature 423, 738-741 (2003)

A.G.W. Cameron, W. Benz, The origin of the moon and the single impact hypothesis. IV. Icarus 92, 204-216 (1991). https://doi.org/10.1016/0019-1035(91)90046-V

R.M. Canup, E. Asphaug, Origin of the Moon in a giant impact near the end of the Earth's formation. Nature 412, 708-712 (2001)

A. Caracausi, G. Avice, P. Bernard, E. Furi, B. Marty, Chondritic xenon in the Earth's mantle. Nature 553, $82-85$ (2016)

J.E. Chambers, Making more terrestrial planets. Icarus 152, 205-224 (2001). https://doi.org/10.1006/ icar.2001.6639

J.E. Chambers, G.W. Wetherill, Making the terrestrial planets: N-body integrations of planetary embryos in three dimensions. Icarus 136, 304-327 (1998). https://doi.org/10.1006/icar.1998.6007 
N. Coltice, M. Moreira, J. Hernlund, S. Labrosse, Crystallization of a basal magma ocean recorded by helium and neon. Earth Planet. Sci. Lett. 308, 193-199 (2011). https://doi.org/10.1016/j.eps1.2011.05.045

M. Ćuk, S.T. Stewart, Making the Moon from a fast-spinning Earth: a giant impact followed by resonant despinning. Science 338, 1047 (2012). https://doi.org/10.1126/science.1225542

R. Dasgupta, H. Chi, N. Shimizu, A.S. Buono, D. Walker, Carbon solution and partitioning between metallic and silicate melts in a shallow magma ocean: implications for the origin and distribution of terrestrial carbon. Geochim. Cosmochim. Acta 102, 191-212 (2013). https://doi.org/10.1016/j.gca.2012.10.011

N. Dauphas, The dual origin of the terrestrial atmosphere. Icarus 165, 326-339 (2003). https://doi.org/10. 1016/S0019-1035(03)00198-2

N. Dauphas, A. Morbidelli, Geochemical and planetary dynamical views on the origin of Earths atmosphere and oceans, in The Atmosphere-History, ed. by D.E. Canfield, J. Farquhar, J.F. Kasting. Treatise on Geochemistry, 2nd edn., vol. 13 (2014). https://doi.org/10.1016/B978-0-08-095975-7.01301-2. Chap. 6.1

N. Dauphas, A. Pourmand, Hf-W-Th evidence for rapid growth of Mars and its status as a planetary embryo. Nature 473, 489-492 (2011). https://doi.org/10.1038/nature10077

L.T. Elkins-Tanton, Linked magma ocean solidification and atmospheric growth for Earth and Mars. Earth Planet. Sci. Lett. 271, 181-191 (2008). https://doi.org/10.1016/j.eps1.2008.03.062

N.V. Erkaev, H. Lammer, L.T. Elkins-Tanton, A. Stökl, P. Odert, E. Marcq, E.A. Dorfi, K.G. Kislyakova, Y.N. Kulikov, M. Leitzinger, M. Güdel, Escape of the martian protoatmosphere and initial water inventory. Planet. Space Sci. 98, 106-119 (2014). https://doi.org/10.1016/j.pss.2013.09.008

E. Füri, D.R. Hilton, S.A. Halldórsson, P.H. Barry, D. Hahm, T.P. Fischer, K. Grönvold, Apparent decoupling of the $\mathrm{He}$ and $\mathrm{Ne}$ isotope systematics of the Icelandic mantle: the role of He depletion, melt mixing, degassing fractionation and air interaction. Geochim. Cosmochim. Acta 74, 3307-3332 (2010). https://doi.org/10.1016/j.gca.2010.03.023

H. Genda, Y. Abe, Survival of a proto-atmosphere through the stage of giant impacts: the mechanical aspects. Icarus 164, 149-162 (2003). https://doi.org/10.1016/S0019-1035(03)00101-5

H. Genda, Y. Abe, Enhanced atmospheric loss on protoplanets at the giant impact phase in the presence of oceans. Nature 433, 842-844 (2005). https://doi.org/10.1038/nature03360

P. Goldreich, Y. Lithwick, R. Sari, Final stages of planet formation. Astrophys. J. 614, 497-507 (2004). https://doi.org/10.1086/423612

R. Grover, J.W. Hardy, The propagation of shocks in exponentially decreasing atmospheres. Astrophys. J. 143, 48 (1966). https://doi.org/10.1086/148476

A.N. Halliday, The origins of volatiles in the terrestrial planets. Geochim. Cosmochim. Acta 105, 146-171 (2013). https://doi.org/10.1016/j.gca.2012.11.015

L.J. Hallis, G.R. Huss, K. Nagashima, G.J. Taylor, S.A. Halldórsson, D.R. Hilton, M.J. Mottl, K.J. Meech, Evidence for primordial water in Earth's deep mantle. Science 350, 795-797 (2015). https://doi.org/10.1126/science.aac4834

B.M.S. Hansen, N. Murray, Migration then assembly: formation of Neptune-mass planets inside 1 AU. Astrophys. J. 751, 158 (2012). https://doi.org/10.1088/0004-637X/751/2/158

C.L. Harper Jr., S.B. Jacobsen, Noble gases and Earth's accretion. Science 273, 1814-1818 (1996). https://doi.org/10.1126/science.273.5283.1814

C. Hayashi, Structure of the solar nebula, growth and decay of magnetic fields and effects of magnetic and turbulent viscosities on the nebula. Prog. Theor. Phys. Suppl. 70, 35-53 (1981)

V.S. Heber, R.A. Brooker, S.P. Kelley, B.J. Wood, Crystal melt partitioning of noble gases (helium, neon, argon, krypton, and xenon) for olivine and clinopyroxene. Geochim. Cosmochim. Acta 71, 1041-1061 (2007). https://doi.org/10.1016/j.gca.2006.11.010

V.S. Heber, H. Baur, P. Bochsler, K.D. McKeegan, M. Neugebauer, D.B. Reisenfeld, R. Wieler, R.C. Wiens, Isotopic mass fractionation of solar wind: evidence from fast and slow solar wind collected by the genesis mission. Astrophys. J. 759, 121 (2012). https://doi.org/10.1088/0004-637X/759/2/121

L.A. Hillenbrand, Disk-dispersal and planet-formation timescales. Phys. Scr. T 130(1), 014024 (2008). https://doi.org/10.1088/0031-8949/2008/T130/014024

M.M. Hirschmann, Constraints on the early delivery and fractionation of Earth's major volatiles from $\mathrm{C} / \mathrm{H}$, $\mathrm{C} / \mathrm{N}$ and $\mathrm{C} / \mathrm{S}$ ratios. Am. Mineral. 101, 540-553 (2016)

M.M. Hirschmann, R. Dasgupta, The H/C ratios of Earth's near-surface and deep reservoirs, and consequences for deep Earth volatile cycles. Chem. Geol. 262, 4-16 (2009)

G. Holland, C.J. Ballentine, Seawater subduction controls the heavy noble gas composition of the mantle. Nature 441, 186-191 (2006). https://doi.org/10.1038/nature04761

G. Holland, M. Cassidy, C.J. Ballentine, Meteorite Kr in Earth's mantle suggests a late accretionary source for the atmosphere. Science 326, 1522 (2009). https://doi.org/10.1126/science.1179518

M. Honda, I. McDougall, Primordial helium and neon in the Earth: a speculation on early degassing. Geophys. Res. Lett. 25, 1951-1954 (1998). https://doi.org/10.1029/98GL01329 
J. Hopp, M. Trieloff, Helium deficit in high-3He/4He parent magmas: predegassing fractionation, not a "helium paradox". Geochem. Geophys. Geosyst. 9, 03009 (2008)

S. Ida, J. Makino, Scattering of planetesimals by a protoplanet-slowing down of runaway growth. Icarus 106, 210 (1993). https://doi.org/10.1006/icar.1993.1167

N.K. Inamdar, H.E. Schlichting, The formation of super-Earths and mini-Neptunes with giant impacts. Mon. Not. R. Astron. Soc. 448, 1751-1760 (2015). https://doi.org/10.1093/mnras/stv030

N.K. Inamdar, H.E. Schlichting, Stealing the gas: giant impacts and the large diversity in exoplanet densities. Astrophys. J. Lett. 817, 13 (2016). https://doi.org/10.3847/2041-8205/817/2/L13

C.R.M. Jackson, S.W. Parman, S.P. Kelley, R.F. Cooper, Constraints on light noble gas partitioning at the conditions of spinel-peridotite melting. Earth Planet. Sci. Lett. 384, 178-187 (2013). https://doi.org/10.1016/j.epsl.2013.09.046

E. Jehin, J. Manfroid, D. Hutsemékers, C. Arpigny, J.-M. Zucconi, Isotopic ratios in comets: status and perspectives. Earth Moon Planets 105, 167-180 (2009). https://doi.org/10.1007/s11038-009-9322-y

S.J. Kenyon, B.C. Bromley, Terrestrial planet formation. I. The transition from oligarchic growth to chaotic growth. Astron. J. 131, 1837-1850 (2006). https://doi.org/10.1086/499807

M.D. Kurz, J. Curtice, D. Fornari, D. Geist, M. Moreira, Primitive neon from the center of the Galápagos hotspot. Earth Planet. Sci. Lett. 286, 23-34 (2009). https://doi.org/10.1016/j.eps1.2009.06.008

S. Labrosse, J.W. Hernlund, N. Coltice, A crystallizing dense magma ocean at the base of the Earth's mantle. Nature 450, 866-869 (2007). https://doi.org/10.1038/nature06355

T. Lebrun, H. Massol, E. ChassefièRe, A. Davaille, E. Marcq, P. Sarda, F. Leblanc, G. Brandeis, Thermal evolution of an early magma ocean in interaction with the atmosphere. J. Geophys. Res., Planets 118, 1155-1176 (2013). https://doi.org/10.1002/jgre.20068

Z.M. Leinhardt, S.T. Stewart, Full numerical simulations of catastrophic small body collisions. Icarus 199, 542-559 (2009). https://doi.org/10.1016/j.icarus.2008.09.013

S.-F. Liu, Y. Hori, D.N.C. Lin, E. Asphaug, Giant impact: an efficient mechanism for the devolatilization of super-Earths. Astrophys. J. 812, 164 (2015). https://doi.org/10.1088/0004-637X/812/2/164

S.J. Lock, S.T. Stewart, S. Mukhopadhyay, Was the atmosphere lost during the Moon-forming giant impact? in Lunar and Planetary Science Conference. Lunar and Planetary Inst. Technical Report, vol. 45 (2014), p. 2843

K. Lodders, B. Fegley, The Planetary Scientist's Companion (1998)

S.G. Love, T.J. Ahrens, Catastrophic impacts on gravity dominated asteroids. Icarus 124, 141-155 (1996). https://doi.org/10.1006/icar.1996.0195

J. Manfroid, E. Jehin, D. Hutsemékers, A. Cochran, J.-M. Zucconi, C. Arpigny, R. Schulz, J.A. Stüwe, I. Ilyin, The CN isotopic ratios in comets. Astron. Astrophys. 503, 613-624 (2009). https://doi.org/10.1051/ 0004-6361/200911859

B. Marty, The origins and concentrations of water, carbon, nitrogen and noble gases on Earth. Earth Planet. Sci. Lett. 313, 56-66 (2012). https://doi.org/10.1016/j.eps1.2011.10.040

B. Marty, G. Avice, Y. Sano, K. Altwegg, H. Balsiger, M. Hässig, A. Morbidelli, O. Mousis, M. Rubin, Origins of volatile elements ( $\mathrm{H}, \mathrm{C}, \mathrm{N}$, noble gases) on Earth and Mars in light of recent results from the ROSETTA cometary mission. Earth Planet. Sci. Lett. 441, 91-102 (2016). https://doi.org/10.1016/ j.epsl.2016.02.031

E. Mazor, D. Heymann, E. Anders, Noble gases in carbonaceous chondrites. Geochim. Cosmochim. Acta 34, 781-824 (1970). https://doi.org/10.1016/0016-7037(70)90031-1

F.M. McCubbin, A. Steele, E.H. Hauri, H. Nekvasil, S. Yamashita, R.J. Hemley, Nominally hydrous magmatism on the Moon. Proc. Natl. Acad. Sci. 107, 11223-11228 (2010). https://doi.org/10.1073/ pnas. 1006677107

W.F. McDonough, Compositional model for the Earth's core, in Treatise on Geochemistry, vol. 2 (2003), p. 568. https://doi.org/10.1016/B0-08-043751-6/02015-6

K.J. Meech, J. Pittichová, A. Bar-Nun, G. Notesco, D. Laufer, O.R. Hainaut, S.C. Lowry, D.K. Yeomans, M. Pitts, Activity of comets at large heliocentric distances pre-perihelion. Icarus 201, 719-739 (2009). https://doi.org/10.1016/j.icarus.2008.12.045

R. Meier, T.C. Owen, D.C. Jewitt, H.E. Matthews, M. Senay, N. Biver, D. Bockelee-Morvan, J. Crovisier, D. Gautier, Deuterium in comet C/1995 O1 (Hale-Bopp): detection of DCN. Science 279, 1707 (1998). https://doi.org/10.1126/science.279.5357.1707

H.J. Melosh, A.M. Vickery, Impact erosion of the primordial atmosphere of Mars. Nature 338, 487-489 (1989). https://doi.org/10.1038/338487a0

H. Mizuno, K. Nakazawa, C. Hayashi, Dissolution of the primordial rare gases into the molten Earth's material. Earth Planet. Sci. Lett. 50, 202-210 (1980). https://doi.org/10.1016/0012-821X(80)90131-4

M. Moreira, Noble gas constraints on the origin and evolution of Earth's volatiles. Geochem. Perspect. 2, 229-403 (2013) 
M. Moreira, S. Charnoz, The origin of the neon isotopes in chondrites and on Earth. Earth Planet. Sci. Lett. 433, 249-256 (2016). https://doi.org/10.1016/j.epsl.2015.11.002

M. Moreira, J. Kunz, C. Allegre, Rare gas systematics in popping rock: isotopic and elemental compositions in the upper mantle. Science 279, 1178 (1998). https://doi.org/10.1126/science.279.5354.1178

M. Moreira, J. Blusztajn, J. Curtice, S. Hart, H. Dick, M.D. Kurz, He and Ne isotopes in oceanic crust: implications for noble gas recycling in the mantle. Earth Planet. Sci. Lett. 216, 635-643 (2003). https://doi.org/10.1016/S0012-821X(03)00554-5

J.L. Mosenfelder, P.D. Asimow, D.J. Frost, D.C. Rubie, T.J. Ahrens, The MgSiO3 system at high pressure: thermodynamic properties of perovskite, postperovskite, and melt from global inversion of shock and static compression data. J. Geophys. Res. 14, 01203 (2009)

S. Mukhopadhyay, Early differentiation and volatile accretion recorded in deep mantle neon and xenon. Nature 486, 101-104 (2012)

M. Nakajima, D.J. Stevenson, Melting and mixing states of the Earth's mantle after the Moon-forming impact. Earth Planet. Sci. Lett. 427, 286-295 (2015). https://doi.org/10.1016/j.eps1.2015.06.023

G. Neukum, B.A. Ivanov, W.K. Hartmann, Cratering records in the inner solar system in relation to the lunar reference system. Space Sci. Rev. 96, 55-86 (2001)

G. Notesco, A. Bar-Nun, T. Owen, Gas trapping in water ice at very low deposition rates and implications for comets. Icarus 162, 183-189 (2003). https://doi.org/10.1016/S0019-1035(02)00059-3

M. Ozima, F.A. Podosek, Noble Gas Geochemistry (2002), p. 286

K. Pahlevan, D.J. Stevenson, Equilibration in the aftermath of the lunar-forming giant impact. Earth Planet. Sci. Lett. 262, 438-449 (2007). https://doi.org/10.1016/j.epsl.2007.07.055

H. Palme, H.S.C. O’Neill, Cosmochemical estimates of mantle composition, in Treatise on Geochemistry, vol. 2, ed. by R.W. Carlson, H.D. Holland, K.K. Turekian (Elsevier, New York, 2014), pp. 1-38. https://doi.org/10.1016/B978-0-08-095975-7.00201-1

R. Parai, S. Mukhopadhyay, The evolution of MORB and plume mantle volatile budgets: constraints from fission Xe isotopes in Southwest Indian Ridge basalts. Geochem. Geophys. Geosyst. 16, 719-735 (2015)

R.O. Pepin, On the origin and early evolution of terrestrial planet atmospheres and meteoritic volatiles. Icarus 92, 2-79 (1991). https://doi.org/10.1016/0019-1035(91)90036-S

R.O. Pepin, D. Porcelli, Origin of noble gases in the terrestrial planets. Rev. Mineral. Geochem. 47, 191-246 (2002)

R.O. Pepin, D. Porcelli, Xenon isotope systematics, giant impacts, and mantle degassing on the early Earth. Earth Planet. Sci. Lett. 250, 470-485 (2006). https://doi.org/10.1016/j.eps1.2006.08.014

S. Péron, M. Moreira, A. Colin, L. Arbaret, B. Putlitz, M.D. Kurz, Neon isotopic composition of the mantle constrained by single vesicle analyses. Earth Planet. Sci. Lett. 449, 145-154 (2016). https://doi.org/10. 1016/j.epsl.2016.05.052

M.K. Petô, S. Mukhopadhyay, K.A. Kelley, Heterogeneities from the first 100 million years recorded in deep mantle noble gases from the Northern Lau back-arc basin. Earth Planet. Sci. Lett. 369, 13-23 (2013). https://doi.org/10.1016/j.eps1.2013.02.012

D. Porcelli, D. Woolum, P. Cassen, Deep Earth rare gases: initial inventories, capture from the solar nebula, and losses during Moon formation. Earth Planet. Sci. Lett. 193, 237-251 (2001). https://doi.org/10. 1016/S0012-821X(01)00493-9

M. Pujol, B. Marty, R. Burgess, Chondritic-like xenon trapped in Archean rocks: a possible signature of the ancient atmosphere. Earth Planet. Sci. Lett. 308, 298-306 (2011). https://doi.org/10.1016/ j.eps1.2011.05.053

Y.P. Raizer, Propagation of a shock wave in an inhomogeneous atmosphere in the direction of decreeing density. Zh. Prikl. Mekham. i Tekhn. Fiz. 4, 49-56 (1964)

A. Raquin, M. Moreira, Atmospheric ${ }^{38} \mathrm{Ar} /{ }^{36} \mathrm{Ar}$ in the mantle: implications for the nature of the terrestrial parent bodies. Earth Planet. Sci. Lett. 287, 551-558 (2009). https://doi.org/10.1016/j.eps1.2009.09.003

S.N. Raymond, R. Barnes, A.M. Mandell, Observable consequences of planet formation models in systems with close-in terrestrial planets. Mon. Not. R. Astron. Soc. 384, 663-674 (2008). https://doi.org/10.1111/j.1365-2966.2007.12712.x

P. Sarda, M. Moreira, T. Staudacher, Rare gas systematics on the southernmost Mid-Atlantic ridge: constraints on the lower mantle and the Dupal source. J. Geophys. Res. 105, 5973-5996 (2000)

H.E. Schlichting, Formation of close in super-Earths and mini-Neptunes: required disk masses and their implications. Astrophys. J. Lett. 795, 15 (2014). https://doi.org/10.1088/2041-8205/795/1/L15

H.E. Schlichting, R. Sari, A. Yalinewich, Atmospheric mass loss during planet formation: the importance of planetesimal impacts. Icarus 247, 81-94 (2015). https://doi.org/10.1016/j.icarus.2014.09.053

H.E. Schlichting, P.H. Warren, Q.-Z. Yin, The last stages of terrestrial planet formation: dynamical friction and the late Veneer. Astrophys. J. 752, 8 (2012). https://doi.org/10.1088/0004-637X/752/1/8 
M. Schönbächler, R.W. Carlson, M.F. Horan, T.D. Mock, E.H. Hauri, Heterogeneous accretion and the moderately volatile element budget of Earth. Science 328, 884 (2010). https://doi.org/10.1126/ science. 1186239

Z.D. Sharp, D.S. Draper, The chlorine abundance of Earth: implications for a habitable planet. Earth Planet. Sci. Lett. 369, 71-77 (2013). https://doi.org/10.1016/j.epsl.2013.03.005

V. Shuvalov, Atmospheric erosion induced by oblique impacts. Meteorit. Planet. Sci. 44, 1095-1105 (2009). https://doi.org/10.1111/j.1945-5100.2009.tb01209.x

V.S. Solomatov, Fluid dynamics of a terrestrial magma ocean, in Origin of the Earth and Moon, ed. by R. Canup, K. Righter (University of Arizona Press, Tucson, 2000), pp. 323-338

T. Staudacher, C.J. Allègre, Recycling of oceanic crust and sediments: the noble gas subduction barrier. Earth Planet. Sci. Lett. 89, 173-183 (1988). https://doi.org/10.1016/0012-821X(88)90170-7

S.T. Stewart, S.J. Lock, S. Mukhopadhyay, Atmospheric loss and volatile fractionation during giant impacts, in Lunar and Planetary Science Conference. Lunar and Planetary Science Conference, vol. 45 (2014), p. 2869

H. Tang, N. Dauphas, ${ }^{60} \mathrm{Fe}-{ }^{60} \mathrm{Ni}$ chronology of core formation in Mars. Earth Planet. Sci. Lett. 390, 264-274 (2014). https://doi.org/10.1016/j.epsl.2014.01.005

C.W. Thomas, Q. Liu, C.B. Agee, P.D. Asimow, R.A. Lange, Multi-technique equation of state for Fe2SiO4 melt and the density of Fe-bearing silicate melts from 0-161 GPa. J. Geophys. Res. 117, 10206 (2012)

M. Trieloff, J. Kunz, D.A. Clague, D. Harrison, C.J. Allègre, The nature of pristine noble gases in mantle plumes Science 288, 1036-1039 (2000)

M. Trieloff, J. Kunz, Isotope systematics of noble gases in the Earth's mantle: possible sources of primordial isotopes and implications for mantle structure. Phys. Earth Planet. Inter. 148, 13-38 (2005)

J.M. Tucker, S. Mukhopadhyay, Evidence for multiple magma ocean outgassing and atmospheric loss episodes from mantle noble gases. ArXiv e-prints (2014)

A.M. Vickery, H.J. Melosh, Atmospheric erosion and impactor retention in large impacts, with application to mass extinctions, in Geological Society of America, vol. 247 (1990), pp. 289-300

R.J. Walker, Highly siderophile elements in the Earth, Moon and Mars: update and implications for planetary accretion and differentiation. Chem. Erde 69, 101-125 (2009). https://doi.org/10.1016/ j.chemer.2008.10.001

R.J. Walker, M.F. Horan, C.K. Shearer, J.J. Papike, Low abundances of highly siderophile elements in the lunar mantle: evidence for prolonged late accretion. Earth Planet. Sci. Lett. 224, 399-413 (2004). https://doi.org/10.1016/j.epsl.2004.05.036

H. Wang, B.P. Weiss, X.-N. Bai, B.G. Downey, J. Wang, J. Wang, C. Suavet, R. Fu, M.E. Zucolotto, Lifetime of the solar nebula constrained by meteorite paleomagnetism. Science 355(6325), 623-627 (2017). https://doi.org/10.1126/science.aaf5043

P.H. Warren, G.W. Kallemeyn, F.T. Kyte, Origin of planetary cores: evidence from highly siderophile elements in Martian meteorites. Geochim. Cosmochim. Acta 63, 2105-2122 (1999). https://doi.org/10.1016/S0016-7037(99)00156-8

S.J. Weidenschilling, D. Spaute, D.R. Davis, F. Marzari, K. Ohtsuki, Accretional evolution of a planetesimal swarm. Icarus 128, 429-455 (1997). https://doi.org/10.1006/icar.1997.5747

Q. Yin, S.B. Jacobsen, K. Yamashita, J. Blichert-Toft, P. Télouk, F. Albarède, A short timescale for terrestrial planet formation from Hf-W chronometry of meteorites. Nature 418, 949-952 (2002). https://doi.org/10.1038/nature00995

R. Yokochi, B. Marty, A determination of the neon isotopic composition of the deep mantle. Earth Planet. Sci. Lett. 225, 77-88 (2004). https://doi.org/10.1016/j.epsl.2004.06.010

K. Zahnle, N. Arndt, C. Cockell, A. Halliday, E. Nisbet, F. Selsis, N.H. Sleep, Emergence of a habitable planet. Space Sci. Rev. 129, 35-78 (2007). https://doi.org/10.1007/s11214-007-9225-z

Y.B. Zel'dovich, Y.P. Raizer, Physics of Shock Waves and High-Temperature Hydrodynamic Phenomena (1967) 